IFT-UAM-CSIC-02-32

hep-th/0208014

\title{
Local models for intersecting brane worlds
}

\author{
Angel M. Uranga 1 \\ I.M.A.F.F. and Instituto de Física Teórica C-XVI \\ Universidad Autónoma de Madrid, 28049 Madrid, Spain
}

\begin{abstract}
We describe the construction of configurations of D6-branes wrapped on compact 3cycles intersecting at points in non-compact Calabi-Yau threefolds. Such constructions provide local models of intersecting brane worlds, and describe sectors of fourdimensional gauge theories with chiral fermions. We present several classes of noncompact manifolds with compact 3-cycles intersecting at points, and discuss the rules required for model building with wrapped D6-branes. The rules to build 3-cycles are simple, and allow easy computation of chiral spectra, RR tadpoles and the amount of preserved supersymmetry. We present several explicit examples of these constructions, some of which have Standard Model like gauge group and three quark-lepton generations. In some cases, mirror symmetry relates the models to other constructions used in phenomenological D-brane model building, like D-branes at singularities. Some simple $\mathcal{N}=1$ supersymmetric configurations may lead to relatively tractable $G_{2}$ manifolds upon lift to M-theory, which would be non-compact but nevertheless yield four-dimensional chiral gauge field theories.
\end{abstract}

\footnotetext{
${ }^{1}$ Angel. Uranga@uam.es
} 


\section{Introduction}

There has been a lot of recent progress in model building of four-dimensional chiral string models with D6-branes wrapped on 3-cycles, mostly centered on phenomenological models [1, 2, 3, 4, 5, 6, 7] (see [8] for earlier work, and [9] for theoretical issues in mainly non-chiral models).

All such models are compact, hence to define the gauge sector one needs to specify the full model, and to some extent one would have to deal with difficult gravitational issues (for instance the issue of the cosmological constant in non-supersymmetric models). Moreover, most models have been worked out in the particular case of six-torus (or orbifolds/orientifolds thereof), with only partial success in extending to Calabi-Yau models [7]. This makes the generality of the results questionable.

On the other hand, other setups like D3-branes located at singularities allowed a more interesting bottom-up approach, where one cooks up a local model (D-branes at a singular point in a non-compact Calabi-Yau) which might subsequently be regarded as a local description for a small region in a full-fledged compactification with fourdimensional gravity. This allows to decouple the gauge sector (which mainly depends on the local structure) from the details of the choice of compactification manifold, and from many difficult (although clearly important) gravitational issues.

It would be highly desirable to have a similar bottom-up approach for models of intersecting D6-branes. This is the task that we undertake in the present paper. Namely we are interested in non-compact Calabi-Yau spaces with compact 3-cycles which intersect at points. D6-branes wrapped on such 3-cycles lead to four-dimensional gauge field theories with chiral fermions arising at intersections, just as in toroidal models. In addition, the models may contain D6-branes wrapped in non-compact 3-cycles; these represent global symmetries from the viewpoint of the four-dimensional theory (their gauge coupling is infinitely suppressed due to the infinite volume) and may become gauged in a fully compact model (depending on the details of the compactification). Such local models may subsequently be embedded in a full-fledged global compactification, with four-dimensional gravity. The important point is that many features of the local D-brane configuration, and hence of the gauge sector of the theory, are insensitive to the details of the compactification. The idea is illustrated in figure 1

In the present paper we present several classes of non-compact Calabi-Yau threefolds with compact 3-cycles intersecting at points. The fact that such local Calabi-Yau spaces exist is clear, for instance from mirror symmetry as follows. As discussed above, D3-branes at singularities lead to localized four-dimensional gauge theories with chiral 
a)

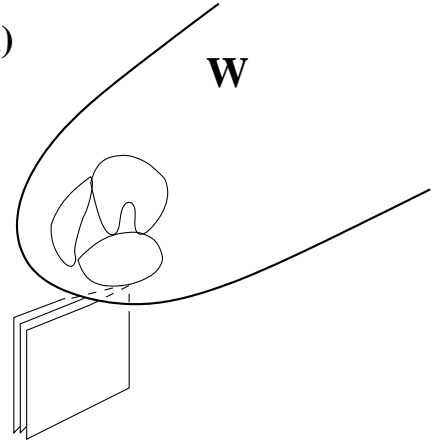

b)

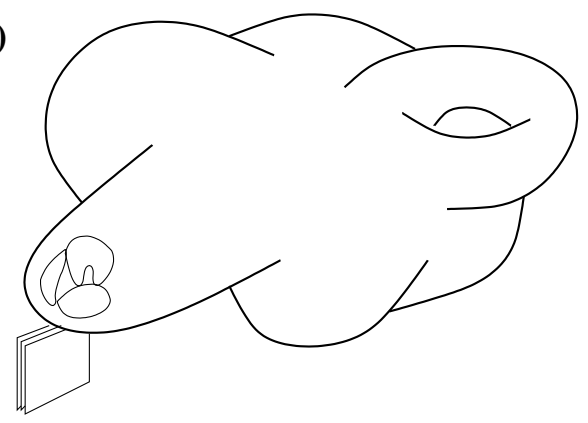

Figure 1: Bottom-up approach to embedding the gauge theory sectors from intersecting D-branes in string compactification. In a first step a) one considers local configurations of D6-branes wrapped on compact 3-cycles in a non-compact space $\mathbf{W}$. The local configuration may be subsequently embedded in a global compactification, step b). Many features of the chiral gauge field theory on the D6-branes are not sensitive to the details of the compactification and depend only on the local geometry $\mathbf{W}$.

fermions. From the viewpoint of D-branes on Calabi-Yau spaces, systems of D3-/D7branes at singularities correspond to the so-called B type branes, namely can be regarded as higher dimensional D-branes wrapped on holomorphic cycles of the CalabiYau $\mathbf{M}$ and carrying holomorphic gauge bundles [10]. Upon application of mirror symmetry, they are mapped to A type branes, in this case D6-branes wrapped on special lagrangian 3-cycles in the mirror geometry $\mathbf{W}$ [10], which is non-compact as well 1. Since mirror symmetry relates configurations yielding the same physics, the final D6-branes in $\mathbf{W}$ must wrap compact 3-cycles intersecting at points, so as to reproduce the four-dimensional gauge theories with chiral fermions obtained from $\mathbf{M}$.

In fact, several interesting Calabi-Yau threefolds $\mathbf{W}$ are familiar from the mirror symmetry literature. However, even though mirror symmetry is a fascinating subject, in this paper we take the viewpoint of studying interesting non-compact threefolds with potential to lead to interesting four-dimensional chiral gauge theories by wrapping D6branes on compact 3-cycles. We are interested in providing a set of rules to compute their spectra for diverse Calabi-Yau constructions, regardless of whether or not their mirror manifolds $\mathbf{M}$ are known 2 .

\footnotetext{
${ }^{1} \mathrm{~A}$ particular case of mirror symmetry (in a global rather than local context) is the T-duality between toroidal models with D9-branes with magnetic world-volume fluxes (see [11, 1, 12] for recent constructions) and toroidal models with D6-branes on 3-cycles; consistently with [13].

${ }^{2}$ However, we would like to point out that some of our results (like for instance the description of
} 
We will describe the basic rules for the construction of compact intersecting 3cycles in two large classes of non-compact Calabi-Yau spaces. Within each class we will present simple examples of models with spectrum similar to the Standard Model, simply to illustrate the richness of the constructions. More detailed search for phenomenological models is left for future work.

One clear advantage of the use of local configurations in model building is that they allow a better handle on the final spectrum of the gauge sector of the theory. A second advantage, from the phenomenological viewpoint, is that models with D6branes wrapped on 3-cycles localized in a small region of the Calabi-Yau allow for a low string scale. Indeed, by enlarging the dimension of the Calabi-Yau which are transverse to the set of D6-branes one may reproduce a large four-dimensional Planck mass, even for low string scales, without enlarging the size of 3-cycles (therefore without the risk of generating too light Kaluza-Klein replicas of Standard Model gauge bosons). This possibility was not allowed in toroidal-like models, since they contain no direction which is transverse to all D6-branes [1].

Finally, some of the wrapped 3-cycles in our models are topologically 3-spheres, hence the corresponding gauge groups do not contain adjoint $\mathcal{N}=1$ chiral multiplets. This is in contrast with toroidal models (and quotients thereof) where the 3-cycles used had non-trivial $b_{1}$ and lead to a number of (phenomenologically undesirable) adjoint matter multiplets.

The paper is organized as follows. In Section 2 we describe a class of non-compact Calabi-Yau threefolds, describe several kinds of compact and non-compact 3-cycles in them, and construct explicit examples of local intersecting brane worlds with D6branes wrapped on 3-cycles. Some of them have gauge sector spectrum close to the Standard Model. In Section 3 we briefly sketch several generalizations of the above construction in more involved threefolds, with a richer structure of compact 3-cycles. Our treatment aims at stating the basic model building rules, and we leave their more systematic exploration for future work. In Section 4 we describe manifolds given by a product of a two-torus times a non-compact two-fold. We describe diverse 3-cycles in such space, and use them to build chiral gauge sectors of intersecting D6-branes. In Section 5 we discuss the construction of some explicit configurations, in certain (nonCY) threefold with topology $\mathbf{T}^{\mathbf{2}} \times \mathbf{T}^{\mathbf{2}} \times \mathbf{Y}$, with $\mathbf{Y}$ a non-compact Riemann surface, and producing just the Standard Model gauge interactions and chiral fermion content.

D6-branes mirror to D7-branes at singularities, section 2.5.2) are not described in the mirror symmetry literature, hence should interest the corresponding readers. 


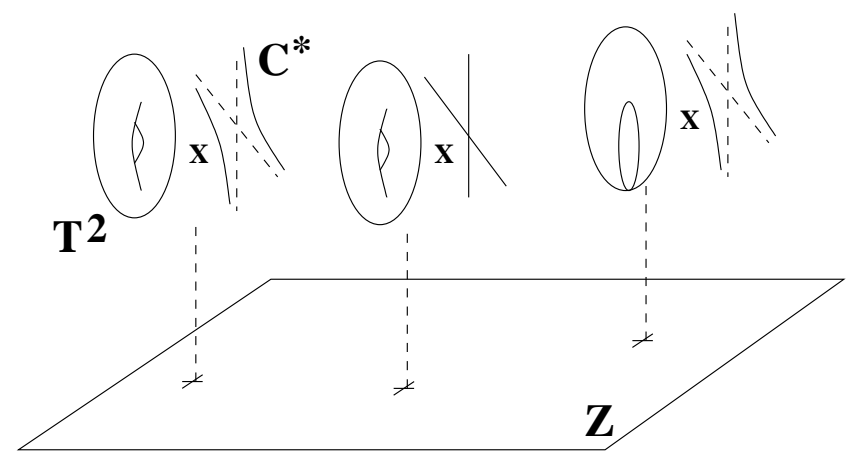

Figure 2: Schematic depiction of the fibration structure of the threefold $\mathbf{W}$. At certain points on the $z$-plane, the $\mathbf{C}^{*}$ or the elliptic fiber degenerate.

In Section 6 we present our concluding remarks.

\section{Local intersecting brane worlds in non-compact threefolds}

In this section we describe a class of non-compact threefolds with compact 3-cycles intersecting at points. They have been studied in [14, 15, 16, 17] in a different context (building on earlier work [18]), so we find it useful to review their basic results.

\subsection{The threefolds}

Let us consider the following class of non-compact Calabi-Yau threefolds, given by the equations

$$
\begin{aligned}
u v & =z \\
y^{2} & =x^{3}+f(z) x+g(z)
\end{aligned}
$$

It corresponds to a non-compact complex plane, parametrized by $z$, over which we have a $\mathbf{C}^{*}$ fibration times an elliptic fibration. The structure of the fibration is sketched in figure 22. Over a generic point $z_{0}$, the $\mathbf{C}^{*}$ fiber is given by $u v=z_{0}$, and contains an $\mathbf{S}^{\mathbf{1}}$ (e.g. for real $z_{0}$, the $\mathbf{S}^{\mathbf{1}}$ is made manifest by introducing $u=x_{1}+i x_{2}, v=x_{1}-i x_{2}$ and taking $x_{i}$ real in $x_{1}^{2}+x_{2}^{2}=z_{0}$ ). At $z=0$ the $\mathbf{C}^{*}$ fiber degenerates to two complex planes, and the $\oint^{1}$ shrinks to zero.

Over a generic point the fiber of the elliptic fibration is a two-torus; the fiber degenerates at points $z_{a}$ which we will label by the $(p, q)$ 1-cycle that collapses. The 
degeneration points and their $(p, q)$ nature depend on the polynomials $f(z), g(z)$ and we specify them in the discussions below. The 1-cycles in the fiber of the elliptic fibration suffer $S L(2, Z)$ monodromies in going around degenerate fibers. Hence, out of each degeneration point of the elliptic fibration, there is a branch cut in the $z$ plane going off to infinity. In crossing (counterclockwise) a branch cut due to a $\left(p_{a}, q_{a}\right)$ degeneration, an $(r, s)$ 1-cycle suffers a monodromy and turns into an $\left(r^{\prime}, s^{\prime}\right)$ one, with

$$
\left(\begin{array}{l}
r \\
s
\end{array}\right) \rightarrow\left(\begin{array}{l}
r^{\prime} \\
s^{\prime}
\end{array}\right)=\left(\begin{array}{cc}
1+p_{a} q_{a} & -p_{a}^{2} \\
q_{a}^{2} & 1-p_{a} q_{a}
\end{array}\right) \cdot\left(\begin{array}{l}
r \\
s
\end{array}\right)=\left(\begin{array}{l}
r \\
s
\end{array}\right)+\left(r q_{a}-s p_{a}\right)\left(\begin{array}{l}
p_{a} \\
q_{a}
\end{array}\right)
$$

The location of these branch cuts is unphysical. However, the $(p, q)$ labels of degenerations themselves may change by moving the branch cuts. Therefore it is convenient to fix a canonical representation for the elliptic fibration; without loss of generality in the discussion of topological properties $\mathrm{B}$, we choose to order the degenerations in counterclockwise circle around $z=0$ in the $z$-plane with branch cuts emanating outwards. We will denote such configuration by

$$
\left(p_{1}, q_{1}\right),\left(p_{2}, q_{2}\right), \ldots,\left(p_{N}, q_{N}\right)
$$

We will not need to move degeneration points through branch cuts ; ; however for completeness we point out that the rules for such crossings are

$$
\begin{aligned}
& \left(p_{i}, q_{i}\right),\left(p_{i+1}, q_{i+1}\right) \rightarrow\left(p_{i+1}, q_{i+1}\right),\left[\left(p_{i}, q_{i}\right)+I_{i, i+1}\left(p_{i+1}, q_{i+1}\right)\right] \\
& \left(p_{i}, q_{i}\right),\left(p_{i+1}, q_{i+1}\right) \rightarrow\left[\left(p_{i+1}, q_{i+1}\right)+I_{i, i+1}\left(p_{i}, q_{i}\right)\right],\left(p_{i}, q_{i}\right)
\end{aligned}
$$

with $I_{i, i+1}=p_{i} q_{i+1}-q_{i} p_{i+1}$.

We will shortly see that this class of threefolds are interesting in that, although they are non-compact still contain a rich structure of compact 3-cycles. Threefolds of this kind (with degenerations specified below) are familiar from the literature on mirror symmetry, since they provide the (local) mirrors of Calabi-Yau threefold singularities given by complex cones over del Pezzo surfaces 0 . The set of $N$ degenerate elliptic fibers

\footnotetext{
${ }^{3}$ Beyond topology, the precise location and nature of the $(p, q)$ degenerations varies over moduli space, or more precisely, over a suitable cover of moduli space (Teichmüller space).

${ }^{4} \mathrm{~A}$ beautiful connection, which we will not exploit in the present paper, relates Picard-Lefschetz transformations (transformations where degeneration points move around branch cuts and return to their original positions) to non-trivial transformation on field theory on D-brane probes (Seiberg dualities). See 19, 20, 21, 16, 22, 17, 23, 24, for further discussions.

${ }^{5}$ For the interested reader, the total space is the anticanonical bundle over the del Pezzo surface. The $k^{\text {th }}$ del Pezzo surfaces can be constructed as the blow up of $\mathbf{P}_{\mathbf{2}}$ at $k$ generic points or the blow up of $\mathbf{P}_{\mathbf{1}} \times \mathbf{P}_{\mathbf{1}}$ at $k-1$ points $(0 \leq k \leq 8)$.
} 
in the mirror $\mathbf{W}$ of the $N^{\text {th }}$ del Pezzo surface is given (in canonical representation) by

$$
(1,0), \ldots,(1,0),(2,-1),(-1,2),(-1,-1)
$$

It is interesting to point out that the complex cone over $\mathbf{P}_{\mathbf{2}}$ is the familiar $\mathbf{C}^{\mathbf{3}} / \mathbf{Z}_{\mathbf{3}}$ orbifold singularity; we will use its mirror, which is the manifold (2.1) with degenerated elliptic fibers $(2,-1),(-1,2),(-1,-1)$, in many of our explicit discussions below. However, extension to other threefolds should be clear.

For future convenience we note that the total monodromy due to the branch cuts of the above set (2.5) of degenerate elliptic fibers is $T^{12-N}$ with

$$
T=\left(\begin{array}{ll}
1 & 1 \\
0 & 1
\end{array}\right)
$$

which leaves the 1 -cycle $(1,0)$ invariant. This total monodromy will turn out important in the construction of certain compact 3-cycles, see Section 2.5.1. However, it is not necessary for consistency, and other elliptic fibration with other sets of degenerate fibers can be studied using similar techniques. In [25] the authors defined and classified isolated configurations of $(p, q)$ degenerations of elliptic fibers, namely those that may be obtained from compact elliptic fibrations by taking a non-compact limit. In order to keep within the non-compact setup one should restrict to those configurations 6 . Any elliptic fibration of this kind would provide a sensible threefold 6 . Even though we will mainly center on the set of degenerations (2.5) for examples, our techniques apply to other examples.

We would like to emphasize that even though these threefolds first appeared in the mirror symmetry context, we are not particularly interested in mirror symmetry. Rather, we use these familiar threefolds to build models of local configurations of D6branes wrapped on intersecting 3-cycles. We expect the results and techniques learnt in this context to extend to other threefolds whose mirror symmetry properties are not particularly interesting/known. However we will make some side remarks on the mirror symmetry interpretation of some of our D6-branes along the way.

\footnotetext{
${ }^{6}$ Even though the techniques in our paper are valid to construct 3-cycles in compact fibrations as well, additional constraints from global tadpole cancellation would complicate the analysis beyond our main interest.

${ }^{7}$ One may be willing to relax the Calabi-Yau condition and consider non-supersymmetric threefolds; to our knowledge there is no easy rule to determine which sets of degenerations give sensible manifolds. One may always start with the Weierstrass form for the elliptic fibration and determine the monodromies due to degenerate fibers.
} 


\subsection{D6-branes on 3-cycles}

\subsubsection{A simple set of compact 3-cycles}

Let us consider a general threefold $\mathbf{W}$ of the above kind, with the elliptic fibration specified by a set of $\left(p_{a}, q_{a}\right)$ degeneration points in canonical representation. We now describe a simple class of compact 3-cycles in this non-compact space, at the topological level. See section 2.3 for a discussion of supersymmetry conditions.

Consider a segment in the $z$-plane that joins (without crossing any branch cut) $z=0$ with the $\left(p_{a}, q_{a}\right)$ degeneration point $z_{a}$. A compact 3-cycle with no boundary is obtained by taking this segment and fibering over it the $\mathbf{S}^{\mathbf{1}}$ in the $\mathbf{C}^{*}$ fibration times the $\left(p_{a}, q_{a}\right)$ 1-cycle in the elliptic fibration, see figures 3 , 自. We will denote such cycle a $\left(p_{a}, q_{a}\right) 3$-cycle. This 3-cycle is topologically a 3-sphere, as follows. Near the pinch of a degenerate fiber at $z_{0}$, an elliptic fibration may be described by $u^{\prime} v^{\prime}=z-z_{0}$. Taking this equation along with the $\mathbf{C}^{*}$ fibration $u v=z$, we obtain $u v-u^{\prime} v^{\prime}=z_{0}$. By changing variables we can recast this as

$$
x_{1}^{2}+x_{2}^{2}+x_{3}^{2}+x_{4}^{2}=z_{0}
$$

Taking e.g. $z_{0}$ real, our 3 -cycle is given by taking real $x_{i}$, which describes an $\mathbf{S}^{\mathbf{3}}$ with size controlled by $z_{0}$, the length of the segment on the $z$ plane.

Two such $\left(p_{a}, q_{a}\right)$ and $\left(p_{b}, q_{b}\right)$ 3-cycles generically intersect at several points, located on the fiber over $z=0$ (where they coincide both in the $z$-plane and in the $\mathbf{C}^{*}$ fibration), see figure 3. Their intersection number when both are identically oriented in $\mathbf{C}^{*}$ and on the $z$-plane (say, both outgoing from $z=0$ ) is given by

$$
I_{a b}=p_{a} q_{b}-q_{a} p_{b}
$$

Hence D6-branes wrapped on these 3-cycles are interesting examples of local configurations of intersecting D6-branes giving four-dimensional gauge field theories with chiral fermions. Explicit examples are discussed in Section 2.5.

This set of 3-cyles is moreover important in that it provides a basis for the compact 3-homology of the manifold W. Hence any other compact 3-cycle can be expressed topologically as a linear combination of 3-cycles in the above set. However, the minimal volume 3-cycle representative of a given homology class may not be a 3 -cycle of the above simple kind, as we describe in the following. 

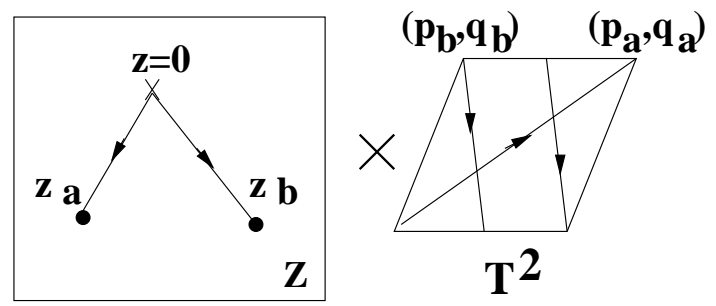

Figure 3: Schematic depiction of the intersection between two compact 3-cycles. The two 3-cycles touch at $z=0$ in the $z$-plane, where they also touch in the $\mathbf{C}^{*}$ fiber since the $\mathbf{S}^{\mathbf{1}}$ shrinks to zero. On the elliptic fiber, the 3 -cycles intersect according to their $(p, q)$ labels.

a)

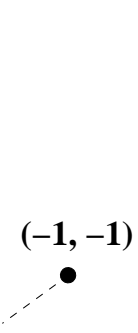

$\bullet(-1,2)$

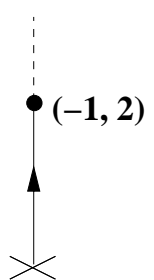

$(2,-1)$

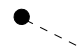

b)
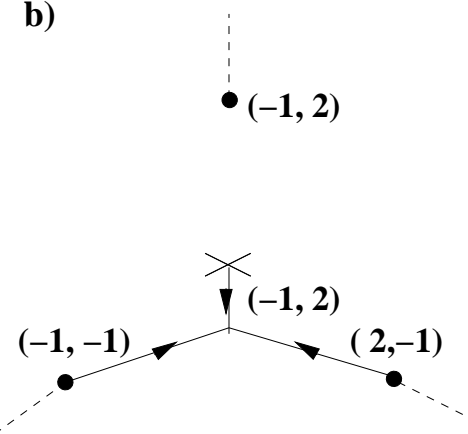

Figure 4: Two examples of different compact 3-cycles. Fig a) shows a compact 3-cycles obtained by fibering over a segment in the $z$-plane a $(p, q)=(-1,2)$ 1-cycle in the elliptic fiber and the $\mathbf{S}^{\mathbf{1}}$ in the $\mathbf{C}^{*}$ fibration. Fig b) shows a compact 3-cycle obtained by fibering suitable $(p, q)$ 1-cycles over segments over a network in the $z$-plane. Note the conservation of $(p, q)$ wrapping numbers across the junction.

\subsubsection{3-cycles from junctions of $(p, q)$ segments}

There are more general compact 3-cycles that the above ones. They can be obtained by taking a network of oriented segments in the $z$-plane, joining at junctions, and with outer legs ending on the $\left(p_{a}, q_{a}\right)$ degenerations or at $z=0$. Over such segments we fiber the $\mathbf{S}_{\mathbf{1}}$ in the $\mathbf{C}^{*}$ fibration and a $(p, q)$ 1-cycle in the elliptic fibration. To obtain a closed 3-cycle the $(p, q)$ label of the segments must be additively conserved at each junction, and segments ending on a degeneration of the elliptic fibration must be of the correct $(p, q)$ kind. One example of a compact 3-cycle from a junction of $(p, q)$ segments is shown in figure 1 .

The rules concerning 3-cycles from junctions are identical to the rules for junctions 


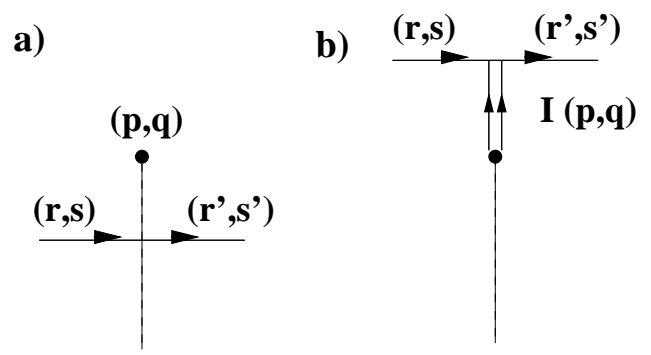

Figure 5: The prong creation process shows the existence of junction 3-cycles in our geometries.

of $(p, q)$ strings in type IIB seven-brane backgrounds 目. In fact our configurations are related by U-duality to type IIB configurations with a set of $\left(p_{a}, q_{a}\right)$ seven-branes (U-dual to our degenerations of the elliptic fibration), one D3-brane (U-dual to the degeneration of the $\mathbf{C}^{*}$ fibration), and $(p, q)$ strings (U-dual to the D6-branes on 3cycles) $\mathrm{Q}$. The topological properties of string junctions have been extensively studied (see e.g. [27, 28, 29, 30, 31, 32, 25]) and we have found those results useful in studying 3-cycles. In fact, some of the above (and below) conventions and phenomena are taken from standard string junction techniques. However we have chosen to briefly re-describe them in our present context.

In analogy with string junctions, it is possible to derive the existence of junction 3 -cycles by the prong creation process [27], illustrated in figures 5 , 6. Consider an $(r, s)$ segment crossing a $(p, q)$ branch cut, hence turning into an $\left(r^{\prime}, s^{\prime}\right)$ segment, see (2.2). Moving the segment across the $(p, q)$ degeneration point, prongs emanating from the latter are created, and the 3 -cycle becomes a junction 3-cycle. The number of prongs outgoing from the degeneration point is given by

$$
I_{(r, s),(p, q)}=r q-p s .
$$

\footnotetext{
${ }^{8}$ These rules have also appeared in the study of networks of type IIB five-branes in seven-brane backgrounds 26.

${ }^{9}$ The duality chain proceeds as follows. We start with a space roughly of the form $\mathbf{C}^{*} \times \mathbf{T}^{\mathbf{2}} \times \mathbf{C}_{\mathbf{Z}}$, with fibered products, with one $\mathbf{C}^{*}$ degeneration, a set of $(p, q)$ elliptic fiber degenerations, and D6branes on 3-cycles made of $(r, s)$ segments. T-dualizing along the three spatial Minkowski directions turns the D6-branes into D3-branes on the same 3-cycle. Further T-duality along the $\mathbf{S}^{\mathbf{1}}$ direction in $\mathbf{C}^{*}$ turns the $\mathbf{C}^{*}$ degeneration into a NS5-brane, and the D3-branes into D2-branes. Subsequent lift to M-theory leads to a geometry $\mathbf{R} \times \mathbf{S}^{\mathbf{1}} \times \mathbf{T}^{\mathbf{2}} \times \mathbf{C}_{\mathbf{Z}}$ with one M5-brane, a set of $(p, q)$ degenerate $\mathbf{T}^{\mathbf{2}}$ fibers, and M2-branes on $(r, s)$ 3-cycles. Upon shrinking $\mathbf{T}^{\mathbf{2}}$ one recovers a IIB model with one D3-brane, a set of $(p, q)$ seven-branes and a network of $(r, s)$ strings.
} 

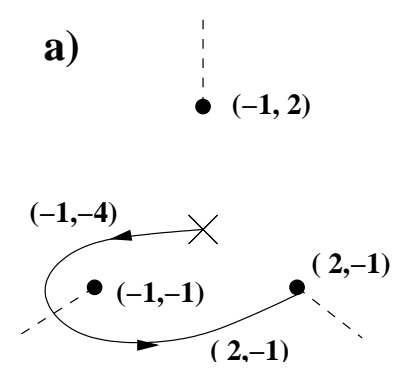

b)

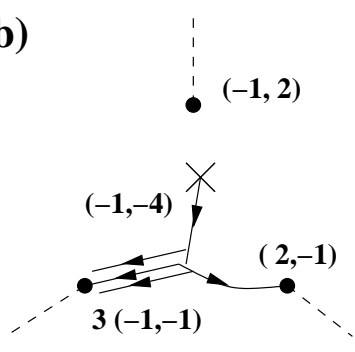

Figure 6: An example of a prong creation process with compact 3-cycles.

This process can be understood from charge conservation on the volume of the $(p, q)$ degeneration, when regarded as an object, or from geometrical considerations as in [19]. It is ultimately related by U-duality to the Hanany-Witten brane creation process [33].

Intersection numbers of junction 3-cycles are easily computed by careful addition (with signs) of intersections of different segments. Notice that two segments crossing away from $z=0$ give zero contribution since since do not intersect on the $\mathbf{C}^{*}$ fiber.

\subsubsection{Non-compact 3-cycles}

It is clear that we have restricted our discussion to compact 3-cycles only for convenience. The setup immediatly allows to construct non-compact 3-cycles by using networks of $(p, q)$ segments with some legs going off to infinity, over which we fiber the $\mathbf{S}^{\mathbf{1}}$ in the $\mathbf{C}^{*}$ fibration times the corresponding $(p, q)$ 1-cycle in the elliptic fibration. In order to obtain consistent 3-cycles, external segments must end at infinity, at a suitable $(p, q)$ degeneration, or at the $\mathbf{C}^{*}$ degeneration point $z=0$.

Intersection numbers between compact and non-compact 3-cycles are computed straightforwardly. However, intersection numbers between non-compact 3-cycles require for their definition some information about asymptotic behaviour (in other words, there may be some intersection number contribution lying at infinity, which would become manifest only upon compactification). To avoid complications, we will not discuss intersection numbers among non-compact 3-cycles.

In this section we would like to introduce a different kind of non-compact 3-cycles, which, instead of wrapping the $\mathbf{S}^{\mathbf{1}}$ in the $\mathbf{C}^{*}$ fibration, span the non-compact direction. At a generic point $z_{0}=r e^{i \theta}$, the latter is given e.g. by taking $u=s e^{i \theta / 2}, v=r / s e^{i \theta / 2}$ in $u v=z_{0}$, with $s \in \mathbf{R}$ parametrizing the non-compact line. The non-compact 3-cycles we are interested in are obtained by taking networks of $(p, q)$ segments (extending 
to infinity in the $z$-plane or not) and fibering the non-compact direction in the $\mathbf{C}^{*}$ fibration, times the corresponding $(p, q)$ 1-cycle in the elliptic fibration. Notice that for these 3-cycles no external segment is allowed to end on the $\mathbf{C}^{*}$ degeneration point, since this would result on a 3-cycle with boundary.

There are two important differences between 3-cycles wrapping the $\mathbf{S}^{\mathbf{1}}$ or the noncompact directions in $\mathbf{C}^{*}$ (momentarily denoted $a$ and $b 3$-cycles, respectively). The first is topological: A non-compact $a$-cycle does not intersect other $a$-cycle (whether compact or not) unless both have segments ending on the $\mathbf{C}^{*}$ degeneration point. In particular, segments crossing away from $z=0$ do not lead to net intersection number, since both cycles are parallel on the $\mathbf{C}^{*}$ fiber. On the other hand, a non-compact $b$ cycle intersects an $a$-cycle at any crossing of segments, with intersection number given by $I_{(p, q),\left(p^{\prime}, q^{\prime}\right)}$. This makes non-compact $b$-cycles more interesting that non-compact a-cycles 10 .

The second important difference concerns the conditions for supersymmetry, so we postpone its discussion until next section.

A last important fact concerning 3-cycles of $b$ kind is the following: when such a 3cycle with label $(p, q)$ crosses over $z=0$, a prong emanating from $z=0$ (with suitable orientation) is created, corresponding to a piece of $a$-cycle with $(p, q)$ label. Creation of this prong is due to the fact that the non-compact direction in $\mathbf{C}^{*}$ intersects the 1-cycle degeneration at $z=0$ (namely, the $\mathbf{S}^{\mathbf{1}}$ in $\mathbf{C}^{*}$ ), and can be shown as previous prong creations processes 11. This fact will be useful in some deformation arguments in section 2.5.2.

\subsection{Supersymmetry and calibrations}

Different D-branes of A type (e.g. D6-branes wrapped on 3-cycles) preserve a common supersymmetry if they calibrate with respect to the same 3 -form $\Omega_{3}$. Namely, if they are special lagrangian (slag) with respect to $\Omega_{3}$. A 3-cyles is special lagrangian if the

\footnotetext{
${ }^{10}$ There are also further sources of intersection which we will not need, but list here for completeness: segments of the $a$ and $b$ kind ending on the same $(p, q)$ degeneration contribute to the intersection number. Similarly, a $(r, s)$ segment crossing the branch cut of a $(p, q)$ degeneration contributes an intersection number $I_{(r, s),(p, q)}$ with a segment ending on the $(p, q)$ degeneration, if they are of $a$ and $b$ kind, or viceversa. This can be seen by moving the $(r, s)$ segment through the degeneration; the newly created prongs do intersect the $(p, q)$ segment.

${ }^{11}$ For instance by recalling that a $\mathbf{C}^{*}$ fibration near a degeneration can be constructed from an elliptic fibration near a degeneration by taking the decompactification limit of the fiber. The $a$ and $b$ cycles in the torus turn into the $a$ and $b$ cycles in the $\mathbf{C}^{*}$
} 
restriction of the Kähler form $J$ vanishes, and if the imaginary part of the restriction of the calibrating 3 -form vanishes $\left(\operatorname{Im}\left(\Omega_{3}\right)=0\right.$ restricted to the 3 -cycle). For the 3-cycles that we consider the former condition is automatically satisfied, hence we just need to impose the latter.

For flat geometries like tori and factorized 3-cycles, the supersymmetry conditions can be stated in terms of simple condition of the angles of the 3-cycle in the different complex planes. Even though our geometries are more involved, it is remarkable that the supersymmetry conditions can be similarly stated as simple conditions on the orientation of the 3 -cycles on the $z$-plane, the $\mathbf{C}^{*}$, and the elliptic fiber.

The geometries at hand are

$$
\begin{aligned}
y^{2} & =x^{3}+f(z) x+g(z) \\
u v & =z
\end{aligned}
$$

We may recast the elliptic fibration in terms of two real variables $x_{1}, x_{2}$ with identifications $x_{1}+i x_{2}=\left(x_{1}+i x_{2}\right)+1$ and $x_{1}+i x_{2}=\left(x_{1}+i x_{2}\right)+\tau$ with $\tau(z)$ the fiber torus period 12 . Then a calibrating 3 -form is

$$
\Omega_{3}=i \frac{d u}{u} \wedge\left(d x_{1}+i d x_{2}\right) \wedge d z
$$

A typical kind of 3-cycles found above is given by

$$
\begin{aligned}
u & =a e^{i \theta} \\
v & =a e^{-i \theta} e^{i \theta_{1}} \\
z & =a^{2} e^{i \theta_{1}} \\
x_{1}+i x_{2} & =(p+\tau q) b
\end{aligned}
$$

parametrized by $a, b, \theta \in \mathbf{R}$. It wraps the $\mathbf{S}^{\mathbf{1}}$ fiber in the $\mathbf{C}^{*}$ fibration, a real line in the $z$-plane, and the $(p, q)$ 1-cycle in the elliptic fiber. Denoting $p+\tau q=L e^{i \theta_{2}}$, we have that $\Omega_{3}$ when restricted to the cycle

$$
\Omega_{3}=-2 L a e^{i\left(\theta_{1}+\theta_{2}\right)} d \theta \wedge d b \wedge d a
$$

The 3-cycles is Slag with respect to the 3-form if $\theta_{1}+\theta_{2}=0$ (if $\theta_{1}+\theta_{2}=\pi$ the wrapped D-brane preserves the opposite supersymmetries, i.e. behaves as the corresponding antibrane). With a suitable choice of conventions, this amounts to requiring that the slope of the base segment in the $z$-plane is adjusted to its $(p, q)$ label.

\footnotetext{
${ }^{12}$ The period is determined from 2.10 ) via the familiar modular invariant j-function, $j(\tau)=\frac{4(24 f)^{3}}{4 f^{3}+27 g^{2}}$
} 
A second kind of 3-cycle is given by

$$
\begin{aligned}
u & =a e^{i \varphi_{0}} \\
v & =b e^{-i \varphi_{0}} e^{i \theta_{1}} \\
z & =a b e^{i \theta_{1}} \\
x_{1}+i x_{2} & =(p+\tau q) c
\end{aligned}
$$

paremetrized by $a, b, c \in \mathbf{R}$. It spans the non-compact direction in $\mathbf{C}^{*}$, a line in the $z$-plane, and wraps the $(p, q)$ 1-cycle in the elliptic fiber. The 3 -form restricted to the 3-cycle gives

$$
\Omega_{3}=i L e^{i\left(\pi / 2+\theta_{1}+\theta_{2}\right)} d a \wedge d b \wedge d c
$$

The 3 -cycle is supersymmetric with respect to that 3 -form if $\theta_{1}+\theta_{2}+\pi / 2=0$. Notice that the 3 -cycle preserves the same susy as the previous one (2.12) if they intersect at angles in $S U(3)$ (taking into account the $\pi / 2$ angle in $\mathbf{C}^{*}$ ) as in [34].

In general we will be happy if every 3-cycle is Slag with respect to some calibrating 3 -form. This simply means that the 3 -cycle is the product of three minimal volume 1-cycles. In particular, they fiber over geodesics (straight lines in the pictures) in the $z$-plane. If all 3-cycles happen to calibrate with a common 3-form (namely their slopes in the $z$-plane are adjusted to their $(p, q)$ labels), an overall $\mathcal{N}=1$ supersymmetry will be preserved. If not, intersections are typically non-supersymmetric; we expect that the corresponding potential open string tachyons may be avoided in some regions of moduli space.

In talking about supersymmetric cycles, an important notion is their moduli space. Let us simply quote the standard result [35] that for a Slag 3-cycle $\Pi$ the (complex) dimension of its moduli space is $b_{1}(\Pi)$. From the field theory viewpoint, this is the number of adjoint matter multiplets of the corresponding gauge group. Hence, Slags which are topologically $\mathbf{S}^{\mathbf{3}}$ lead to no adjoint matter, while Slags which are topologically $\mathbf{T}^{\mathbf{3}}$ have three adjoint chiral multiplets.

We conclude by mentioning that in most arguments above and to follow, involving only topological properties, we will not care about susy at all, even for a single set of branes. Hence we drop the Slag condition for topological issues.

\subsection{Tadpole cancelation}

In a compact threefold $\mathbf{W}$, the $\mathrm{RR}$ tadpole cancellation conditions for a set of $N_{a} \mathrm{D} 6_{a^{-}}$ branes wrapped on 3-cycles with homology classes $\left[\Pi_{a}\right]$ are obtained from consistency 
of the equations of motion of the RR 7 -form $C_{7}$. From the action

$$
S_{C_{7}}=\int_{M_{4} \times W} H_{8} \wedge * H_{8}+\sum_{a} N_{a} \int_{M_{4} \times \Pi_{a}} C_{7}
$$

(where $H_{8}$ is the field strength tensor for $C_{7}$ ) the equations of motion read

$$
d H_{8}=\sum_{a} N_{a} \delta\left(\Pi_{a}\right)
$$

with $\delta(\Pi)$ is a bump 3-form with support on the 3-cycle $\Pi$. This equation in homology gives an integrability condition (Gauss' law)

$$
\left[\Pi_{\mathrm{tot}}\right]=\sum_{a} N_{a}\left[\Pi_{a}\right]=0
$$

In a non-compact space, RR flux may escape to infinity, so the total homology class need not vanish. Rather, charge associated to homology classes which may be taken away to infinity by deformation should be allowed. Such homology classes correspond to cycles which do not intersect any localized 3-cycle (and hence are not in principle topologically obstructed to be carried away to infinity, at least for sufficiently non-compact manifolds [36]). Therefore the RR tadpole conditions for non-compact threefolds read

$$
\left[\Pi_{\mathrm{tot}}\right] \cdot\left[\Sigma_{i}\right]=0
$$

where $\left[\Sigma_{i}\right]$ are a basis of compact 3-cycles. This automatically guarantees cancellation of cubic non-abelian anomalies 13 , as can be easily checked.

Since a general discussion of the contribution of different sets of branes to the tadpoles is involved, we postpone the detailed computation of tadpoles to the explicit examples below. For the time being, it is enough to state that the conditions boil down to requiring cancellation of gauge anomalies in the generic gauge theory from D6-branes wrapped on the basic compact 3-cycles 14 (as expected from other discussions in noncompact setups where RR tadpoles and anomalies were shown to be equivalent [36]). Notice that in embedding the local model into a global compact one, the remaining tadpole may be saturated by adding branes in the correct homology class. From the viewpoint of the gauge group in the non-compact case, this would be a hidden nonintersecting brane, leaving the local gauge sector unchanged (although in the compact setup the brane may become visible, if it is charged under groups of previously noncompact branes, since the latter become gauged upon compactification).

\footnotetext{
${ }^{13}$ It also guarantees that mixed $U(1)$ - non-abelian anomalies cancel by a Green-Schwarz mechanism, involving scalars arising from integrals of the RR 3-form over 3-cycles. Its derivation is given in 2], section 3.2.2, where it was already announced that its validity was beyond the toroidal setup.

${ }^{14} \mathrm{By}$ this we mean requiring the number of fundamentals and antifundamentals to be equal for all nodes in the quiver gauge theory, regardless of the number of branes associated to such node.
} 

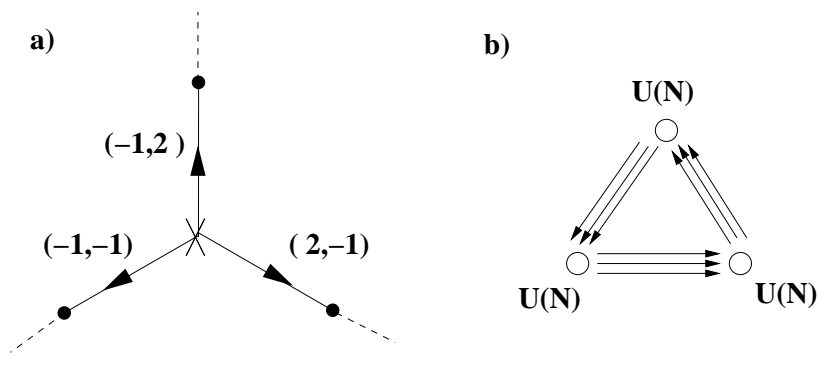

Figure 7: Configuration of D6-branes wrapped on compact 3-cycles in a RR tadpole free fashion.

\subsection{Some examples of model building}

In this section we describe some simple examples of four-dimensional chiral gauge theories which can be obtained using the above threefolds.

\subsubsection{A simple example with matter triplication}

Consider the threefold $\mathbf{W}$ defined by (2.1) with elliptic fiber degenerations

$$
(2,-1),(-1,2),(-1,-1)
$$

Consider a configuration of $N$ D6-branes wrapping each of the three $\mathbf{S}^{\mathbf{3}}$ basic 3-cycles obtained from segments stretching from $z=0$ to the $(p, q)$ degeneration points of the elliptic fibration, figure 7 a.

Let us denote $\left[\Sigma_{(p, q)}\right]$ the homology class of the corresponding 3-cycle. It is easy to check that this configuration satisfies cancellation of RR tadpoles. Specifically, the sum of the three basic 3-cycles is homologous to a 3-cycle which can be taken away to infinity. The latter 3-cycle is given topologically by a closed path around the origin in the $z$-plane over which we fiber the $\mathbf{S}^{\mathbf{1}}$ in the $\mathbf{C}^{*}$ fibration times a 1-cycle in the elliptic fibration, with $(p, q)$ labels as in figure 8a. Note that the existence of this 3-cycle is possible from the fact that the total $S L(2, Z)$ monodromy in the elliptic fibration is $T^{9}$, see discussion around (2.6). The 3 -cycle is topologically a 3 -torus, so it has a three complex dimensional moduli space (corresponding to motion in the three transverse directions, plus turning on Wilson lines).

Figures $8 \mathrm{a}, \mathrm{b}, \mathrm{c}$ illustrate the continuous process, involving brane creation, which connects the homological sum of the three $\mathbf{S}^{\mathbf{3}}$ 's to the final 3-cycle, thus proving RR tadpole cancellation in the setup. Hence, we can write the equation

$$
\left[\Sigma_{(-1,-1)}\right]+\left[\Sigma_{(2,-1)}\right]+\left[\Sigma_{(-1,2)}\right]=0
$$



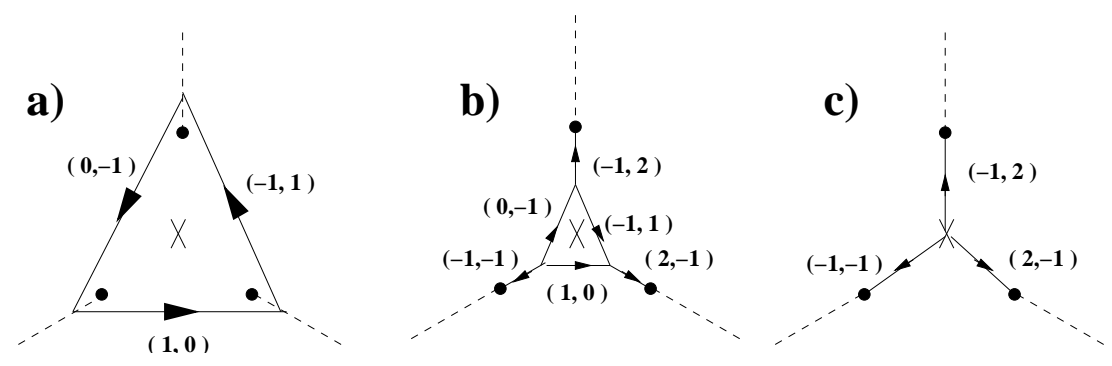

Figure 8: Continuous process showing the total configuration of D6-branes in figure 7 is homologous to a cycle which can be taken to infinity.

modulo a homology element without intersections with the compact 3-cycles (namely, the homology class of the $\mathbf{T}^{\mathbf{3}}$ 3-cycle).

It is interesting to note that for a suitable choice of location of the degeneration points, the above configurations are $\mathcal{N}=1$ supersymmetric, i.e. satisfy the Slag condition in section 2.3 for a common calibrating form. Namely, the slopes of different segments in the $z$-plane are adjusted to their $(p, q)$ labels. As usual, deformations of complex structure away from that point in moduli space result in the generation of Fayet-Iliopoulos terms, potentially triggering brane recombination and gauge symmetry breaking. For simplicity we choose the supersymmetric configuration, as shown in the figures.

The intersection numbers are easily computed from our rules

$$
\left[\Sigma_{(-1,-1)}\right] \cdot\left[\Sigma_{(2,-1)}\right]=\left[\Sigma_{(2,-1)}\right] \cdot\left[\Sigma_{(-1,2)}\right]=\left[\Sigma_{(-1,2)}\right] \cdot\left[\Sigma_{(-1,-1)}\right]=3
$$

The spectrum of the four-dimensional theory on the D6-branes is

$$
\begin{array}{cc}
\text { Gauge group } & U(N) \times U(N) \times U(N) \\
\mathcal{N}=1 \text { Ch. Mult. } & 3(\square, \bar{\square}, 1)+3(1, \square, \bar{\square})+3(\bar{\square}, 1 \square)
\end{array}
$$

The spectrum can be encoded in the quiver diagram 15 in figure $7 \mathrm{~b}$. Even though it is not realistic, it is a good simple example of a local model for an intersecting brane world.

\section{Mirror symmetry interpretation}

This kind of D6-brane configuration has been quite studied in the mirror symmetry literature for threefolds $\mathbf{W}$ corresponding to mirrors of complex cones over del Pezzo

\footnotetext{
${ }^{15} \mathrm{~A}$ quiver is just a picture encoding the chiral spectrum of a theory, with nodes representing gauge factors and arrows representing bi-fundamental matter, see e.g. [37, 38, 39, 40, 41.
} 
surfaces [14, 15, 16, 17]. In fact the set of D6-branes we have built maps to D3-branes sitting at the singularity $\mathbf{M}$. Moreover, the D6-brane wrapped on the $\mathbf{T}^{\mathbf{3}}$ able to move off to infinity is mirror to a D3-brane in $\mathbf{M}$ away from the singular point. The continuous deformation of 3-cycles in figure 14 is mirror to moving a D3-brane off the singularity.

The explicit threefold above is mirror to the $\mathbf{C}^{\mathbf{3}} / \mathbf{Z}_{\mathbf{3}}$ orbifold singularity, so the spectrum on the D6-branes wrapped on the three $\mathbf{S}^{\mathbf{3}}$ 's matches that of a set of D3branes (in $N$ copies of the regular representation of $\mathbf{Z}_{\mathbf{3}}$ ) at the singularity 37, 38, 39, 40, 42. It is amusing to note that the superpotential of the configuration, which arises via open string world-sheet instantons in the D6-brane configuration, should match the familiar result from D3-branes at singularities [38, 39, 40].

It is interesting to note that, at the point in complex structure moduli space where the D6-brane configuration is supersymmetric, the geometry $\mathbf{W}$ has a $\mathbf{Z}_{\mathbf{3}}$ symmetry. It corresponds to the symmetry

$$
\begin{aligned}
z & \rightarrow e^{2 \pi i / 3} z \\
\left(\begin{array}{l}
p \\
q
\end{array}\right)^{z} & \rightarrow\left(\begin{array}{cc}
-1 & -1 \\
1 & 0
\end{array}\right)\left(\begin{array}{l}
p \\
q
\end{array}\right)
\end{aligned}
$$

namely a rotation on the $z$-plane times an order three $S L(2, Z)$ action on the elliptic fiber (this is simpy an order three geometric action $x_{1}+i x_{2} \rightarrow e^{2 \pi i / 3}\left(x_{1}+i x_{2}\right)$ on the complex coordinate of the elliptic fiber). This is the mirror of the point in Kähler moduli space of $\mathbf{M}$ that corresponds to the CFT orbifold, and the symmetry is the so-called $\mathbf{Z}_{\mathbf{3}}$ quantum symmetry of the orbifold $\mathrm{CFT}$, not visible in classical geometry but present in the $\alpha^{\prime}$-exact theory 16 .

It is amusing to compare and match the different ingredients in both mirror constructions (for instance, the RR tadpoles cancellation conditions, the cancellation of mixed $U(1)$ - non-abelian anomalies via the Green-Schwarz mechanism, 43] vs. [2], etc), which had been independently derived for D-branes at singularities and D-branes wrapped on intersecting cycles. Thus seemingly different mechanisms end up related by mirror symmetry. Leaving the discussion as an exercise for the interested reader, we turn to construct further models.

Finally we mention that quiver diagrams providing the spectra and superpotentials for other similar configurations of D6-branes on compact 3-cycles in other threefolds

\footnotetext{
${ }^{16}$ There is also a less interesting symmetry, the symmetry between the three complex planes in $\mathbf{C}^{\mathbf{3}} / \mathbf{Z}_{\mathbf{3}}$ (which also relates the three bi-fundamental chiral multiplets on brane probes). A discrete subgroup of it seems to be also geometrically realized in the manifold $\mathbf{W}$ as a shift of the elliptic fiber (which exchanges the different intersection among 3-cycles).
} 
W (mirror to other cones over del Pezzo surfaces) may be found in e.g. [15]. Instead of reviewing this known material, we turn to the discussion of new models, including D6-branes on non-compact 3-cycles.

\subsubsection{A Standard Model -like example}

\section{Some non-compact 3-cycles}

In this section we would like to consider the same threefold as above, but include D6-branes on non-compact 3-cycles. They will correspond to global symmetries from the viewpoint of the four-dimensional gauge field theory.

Clearly there is a lot of freedom in choosing non-compact 3-cycles. To somehow constrain it, we choose to consider 3 -cycles that preserve the same $\mathcal{N}=1$ supersymmetry as the above D6-brane configuration (at the $\mathbf{Z}_{\mathbf{3}}$ symmetric point in complex structure moduli space). We consider for instance the non-compact 3-cycles obtained by fibering the non-compact direction in $\mathbf{C}^{*}$ and the corresponding $(p, q)$ 1-cycles in the elliptic fiber over the lines shown in figure 9. Using the conditions in section 2.3, one may check that these 3 -cycles are supersymmetric, if one chooses the slopes in the $z$-planes as in figure 9 .

Let us denote $\left[\Lambda_{(p, q)}^{ \pm}\right]$the homology classes of the non-compact $(p, q)$ 3-cycles to the right (resp. left), as one moves counterclokwise, of the compact 3-cycle which they do not intersect. Hence, the classes of the 3-cycles in figure $9 \mathrm{a}, \mathrm{b}, \mathrm{c}$ are $\left[\Lambda_{(-1,1)}^{ \pm}\right],\left[\Lambda_{(0,-1)}^{ \pm}\right]$, $\left[\Lambda_{(1,0)}\right]^{ \pm}$

The intersection numbers of these non-compact 3-cycles with the basic compact 3-cycles are

$$
\begin{aligned}
& {\left[\Lambda_{(-1,1)}^{+}\right] \cdot\left[\Sigma_{(2,-1)}\right]=1 \quad ; \quad\left[\Lambda_{(-1,1)}^{+}\right] \cdot\left[\Sigma_{(-1,-1)}\right]=\left[\Lambda_{(-1,1)}^{+}\right] \cdot\left[\Sigma_{(-1,2)}\right]=0} \\
& {\left[\Lambda_{(-1,1)}^{-}\right] \cdot\left[\Sigma_{(-1,2)}\right]=-1 \quad ; \quad\left[\Lambda_{(-1,1)}^{-}\right] \cdot\left[\Sigma_{(-1,-1)}\right]=\left[\Lambda_{(-1,1)}^{-}\right] \cdot\left[\Sigma_{(2,-1)}\right]=0(2.25)}
\end{aligned}
$$

while the remaining can be determined from the $\mathbf{Z}_{\mathbf{3}}$ symmetry (2.24).

Now we would like to consider a general configuration of D6-branes wrapped on the compact 3-cycles $\left[\Sigma_{(p, q)}\right]$ and on these non-compact 3-cycles. The spectrum of the resulting four-dimensional gauge field theory can be encoded in the quiver diagram fig 10a), where outer nodes correspond to global symmetry groups associated to noncompact D6-branes.

\section{Interesection numbers and RR tadpoles}

In order to build consistent configurations these wrapped D6-branes must be combined consistently with cancellation of localized RR tadpoles. As discussed above, this 

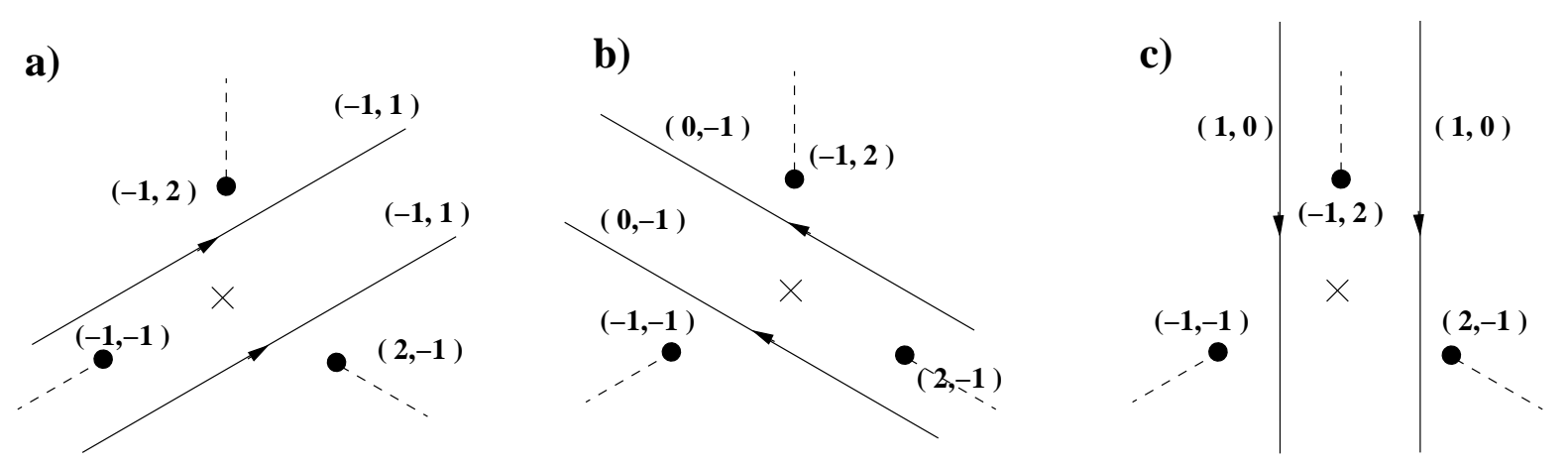

Figure 9: Several useful examples of non-compact 3-cycles.
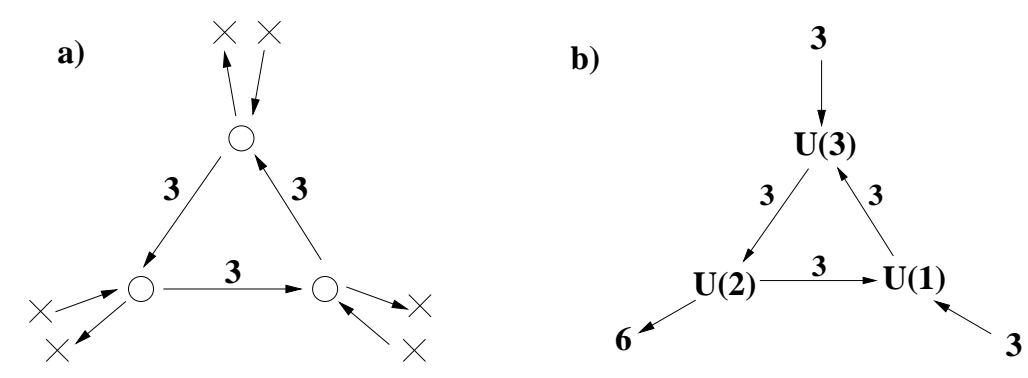

Figure 10: Figure a) shows the quiver diagram for the generic gauge theory sector on a set of D6-branes wrapped on the basic compact $(p, q) 3$-cycles and on the non-compact 3-cycles in figure 9. Figure b) shows the quiver diagram encoding a chiral gauge theory constructed using a particular (tadpole free) choice of wrapped D6-branes; the spectrum is remarkably close to that of the Standard Model. 
amounts to requiring the numbers of fundamentals and antifundamentals to be equal for any internal node in the quiver in figure 10a. Equivalently, we may take the total homology class obtained by wrapping $N_{(p, q)}, M_{(p, q)}^{ \pm}$D6-branes on the 3-cycles $\left[\Sigma_{(p, q)}\right]$, $\left[\Lambda_{(p, q)}^{ \pm}\right]$, and impose the condition (2.19) to obtain

$$
\begin{array}{r}
3 N_{(-1,-1)}-3 N_{(2,-1)}+M_{(0,-1)}^{+}-M_{(-1,1)}^{-}=0 \\
3 N_{(2,-1)}-3 N_{(-1,2)}+M_{(1,0)}^{+}-M_{(0,-1)}^{-}=0 \\
3 N_{(-1,2)}-3 N_{(-1,-1)}+M_{(-1,1)}^{+}-M_{(1,0)}^{-}=0
\end{array}
$$

For completeness, we would like to present a more geometrical viewpoint on the contribution of non-compact 3-cycles to localized RR tadpoles. Using the intersection numbers with the compact 3-cycles, it is easy to obtain certain relations between homology classes, which hold in compactly supported homology (that is, up to homology elements non-intersecting the compact 3-cycles). For instance

$$
\begin{gathered}
{\left[\Sigma_{(-1,-1)}\right]+\left[\Sigma_{(2,-1)}\right]+\left[\Sigma_{(-1,2)}\right]=0} \\
3\left[\Lambda_{(-1,1)}^{+}\right]+3\left[\Lambda_{(0,-1)}^{-}\right]=\left[\Sigma_{(-1,2)}\right] ; 3\left[\Lambda_{(1,0)}^{+}\right]+3\left[\Lambda_{(1,0)}^{-}\right]=-\left[\Sigma_{(-1,2)}\right] \\
3\left[\Lambda_{(0,-1)}^{+}\right]+3\left[\Lambda_{(1,0)}^{-}\right]=\left[\Sigma_{(-1,-1)}\right] ; 3\left[\Lambda_{(-1,1)}^{+}\right]+3\left[\Lambda_{(-1,1)}^{-}\right]=-\left[\Sigma_{(-1,-1)}\right] \\
3\left[\Lambda_{(1,0)}^{+}\right]+3\left[\Lambda_{(-1,1)}^{-}\right]=\left[\Sigma_{(2,-1)}\right] ; \quad 3\left[\Lambda_{(-1,1)}^{+}\right]+3\left[\Lambda_{(-1,1)}^{-}\right]=-\left[\Sigma_{(2,-1)}\right]
\end{gathered}
$$

Alternatively, such relations can be derived by a deformation argument, shown in figures 8, 11, 12 for the relations in the first and second lines.

Use of relations of this kind would allow a re-derivation of (2.26). We prefer instead to use them directly in the example below.

\section{The SM like example}

We would like to apply the above ingredients in the construction of a local intersecting brane world model with semi-realistic spectrum. A simple and very interesting example is provided by combining compact and non-compact 3-cycles of the above kinds in a RR tadpole-free fashion as indicated in figure 10 b). The numbers in the outer nodes denote the number of D6-branes wrapped in the corresponding non-compact 3-cycles (these need not be overlapping, hence the global symmetry is in general abelian, with non-abelian enhancement in non-generic situations of overlapping branes).

One easily verifies that the D6-brane numbers satisfy the localized RR tadpole cancellation equations (2.26). Equivalently, one may use the relations (2.28) to show that the total localized tadpole vanishes

$$
\begin{gathered}
3\left[\Sigma_{(-1,2)}\right]+2\left[\Sigma_{(-1,-1)}\right]+\left[\Sigma_{(2,-1)}\right]+6\left[\Lambda_{(1,0)}^{+}\right]+3\left[\Lambda_{(-1,1)}^{-}\right]+3\left[\Lambda_{(1,0)}^{-}\right]= \\
=2\left[\Sigma_{(-1,2)}\right]+2\left[\Sigma_{(-1,-1)}\right]+2\left[\Sigma_{(2,-1)}\right]=0
\end{gathered}
$$



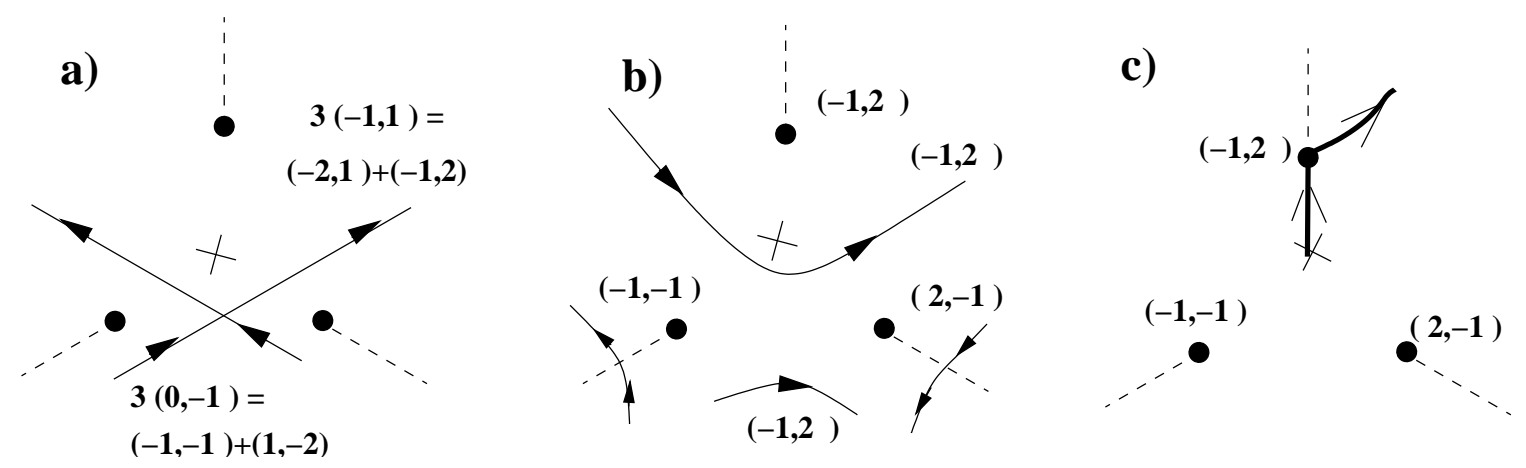

Figure 11: Geometric derivation of the relation $3\left[\Lambda_{(-1,1)}^{+}\right]+3\left[\Lambda_{(0,-1)}^{-}\right]=\left[\Sigma_{(-1,2)}\right]$. We start in a) from the two sets of non-compact $\Lambda$ 3-cycles; after splitting their 1-cycle fiber in the elliptic fibration, recombining the $(-1,2)$ segments, and moving $(p, q)$ segments across $(p, q)$ degenerations, without prong creation, we reach b); moving the remaining $(-1,2)$ segment across the $\mathbf{C}^{*}$ degeneration, with prong creation, (we use thickness to signal that the prong wraps the $\mathbf{S}^{\mathbf{1}}$ in $\mathbf{C}^{*}$, as opposed to the original cycle) leaves a compact 3 -cycle $\left[\Sigma_{(-1,2)}\right]$ plus non-compact 3-cycles not intersecting the compact ones.
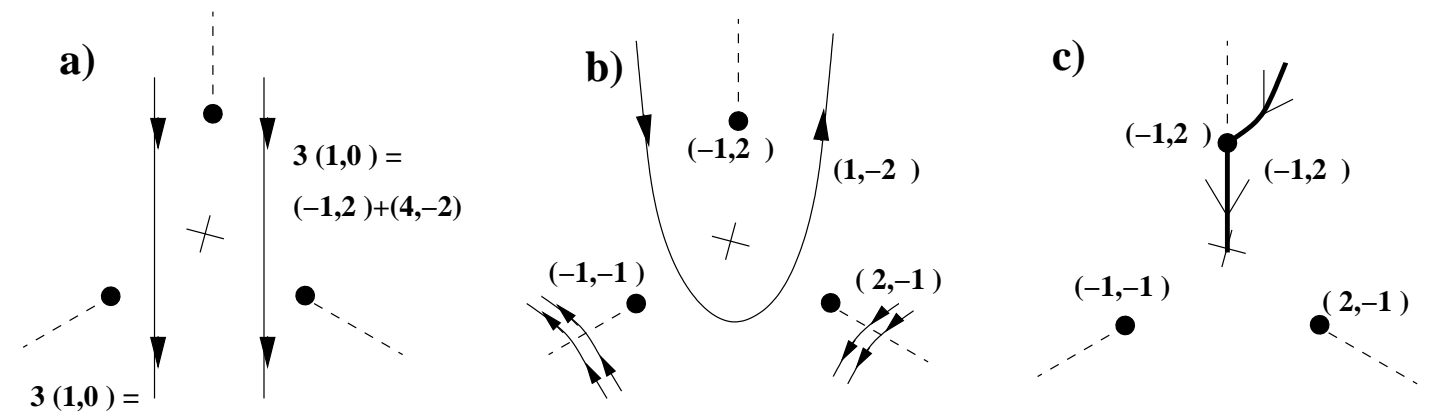

Figure 12: Geometric derivation of the relation $3\left[\Lambda_{(1,0)}^{+}\right]+3\left[\Lambda_{(1,0)}^{-}\right]=-\left[\Sigma_{(-1,2)}\right]$. We start in a) from the two sets of non-compact $\Lambda$ 3-cycles; after splitting their 1-cycle fiber in the elliptic fibration, recombining the $(1,-2)$ segments, and moving $(p, q)$ segments across $(p, q)$ degenerations, without prong creation, we reach b); moving the remaining $(1,-2)$ segment across the $\mathbf{C}^{*}$ degeneration, with prong creation, leaves a compact 3 -cycle $-\left[\Sigma_{(-1,2)}\right]$ plus non-compact 3-cycles not intersecting the compact ones. 
The initial four-dimensional gauge group is $U(3) \times U(2) \times U(1)$, but is is easy to check that two linear combinations of the $U(1)$ 's are anomalous (with anomaly cancelled by the Green-Schwarz mechanism) and get massive due to the $B \wedge F$ coupling 17 . A third linear combination

$$
Q_{Y}=-\left(\frac{1}{3} Q_{3}+\frac{1}{2} Q_{2}+Q_{1}\right)
$$

(with $Q_{i}$ the $U(1)$ generator of the corresponding $U(N)$ factor) does not couple to any four-dimensional B-field and hence remains massless. The generator $Q_{Y}$ plays the role of hypercharge in the model, as can be checked from the full chiral multiplet spectrum

$$
\begin{gathered}
S U(3) \times S U(2) \times U(1)_{Y} \\
3(3,2)_{1 / 6}+3(\overline{3}, 1)_{-2 / 3}+3(\overline{3}, 1)_{1 / 3}+ \\
+3(1,2)_{1 / 2}+3(1,1)_{1}+6(1,2)_{-1 / 2}
\end{gathered}
$$

which correctly reproduces left-handed quarks, right-handed quarks, leptons and three pairs of vector-like higgs multiplets.

Notice that we have ignored states arising from intersections among non-compact 3-cycles, mainly because they are uncharged under the four-dimensional gauge group in the non-compact setup. Upon compactification, the original non-compact cycles also provide four-dimensional gauge interacions; however compactification will in general also influence the number of intersection among originally non-compact 3-cycles, so it is not too meaningful to study these states in a compactification-independent setup.

Clearly, many phenomenological features of the above model could be mentioned here. However we prefer to move on and simply mention that the main features are similar to those already mentioned in [2] 18. However, notice that, as mentioned in the introduction, the present model avoids the problems in lowering the string scale present in the toroidal setup.

It would be interesting to study the possibilities of model building in models with non-trivial NS-NS and RR flux. As discussed in [45], suitable configurations of fluxes may contribute to RR tadpoles, hence replacing some of the D-branes. This may lead

\footnotetext{
${ }^{17}$ Following [2], these are given in our case by $\sum_{a} N_{\left(p_{a}, q_{a}\right)} B_{a} F_{a}$, with $B_{a}=\int_{\Sigma_{\left(p_{a}, q_{a}\right)}} C_{5}$.

${ }^{18} \mathrm{An}$ important particular feature of this model is that it presents gauge coupling unification at the string scale if we sit at the $\mathbf{Z}_{\mathbf{3}}$ symmetric point. Hence unification may be considered accidental (not generic over moduli space), or barely natural (since there is an enhanced symmetry at such point in moduli space. We would like to note in passing that by the mirror symmetry relation below, the same statements can be made concerning the models in 44].
} 


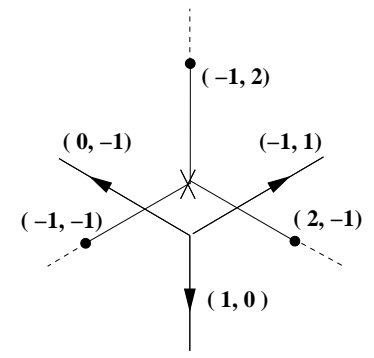

Figure 13: One of the 3-cycles, $\Pi_{(1,0)}$, on which wrapped D6-branes reproduce the mirror of D7-branes at the $\mathbf{C}^{\mathbf{3}} / \mathbf{Z}_{\mathbf{3}}$ singularity. The remaining two are obtained by applying the $\mathbf{Z}_{\mathbf{3}}$ geometric symmetry.

to an interesting mechanism to potentially getting rid of unwanted gauge factors and/or matter multiplets. We leave this interesting possibility for future work.

\section{Mirror symmetry, and further models}

Given that the threefold we have used is mirror to the $\mathbf{C}^{\mathbf{3}} / \mathbf{Z}_{\mathbf{3}}$ orbifold singularity, one may wonder whether the above Standard Model D-brane configuration has a construction in terms of $\mathbf{D}$-branes at the $\mathbf{Z}_{\mathbf{3}}$ singularity. Phenomenological model-building with such D-branes was studied in [44 (see also [46), and it is interesting to notice that the above model was not found. The key point is that the non-compact D-branes used in [4], namely D7-branes, even though preserved $\mathcal{N}=1$ supersymmetry, do not coincide with the mirrors of our non-compact D6-branes.

It is a simple exercise to construct D6-branes on non-compact 3-cycles which are mirror to D7-branes on the $\mathbf{Z}_{\mathbf{3}}$ singularity. One such 3-cycle, denoted $\Pi_{(1,0)}$, is shown in figure 13 , while the remaining two (denoted $\left.\Pi_{(-1,1)}, \Pi_{(0,-1)}\right)$ are obtained through action of the $\mathbf{Z}_{\mathbf{3}}$ geometric symmetry 19.

The contribution of such 3-cycles to localized RR tadpoles are easily computed by checking intersection numbers with the basic compact 3-cycles, or by a deformation argument as shown in figure 14. The corresponding homology relation may be written as

$$
3\left[\Pi_{(-1,1)}\right]=\left[\Sigma_{(-1,-1)}\right]
$$

\footnotetext{
${ }^{19}$ One may wonder which holomorphic 4-cycle the D7-brane is wrapping in $\mathbf{C}^{\mathbf{3}} / \mathbf{Z}_{\mathbf{3}}$. To some extent this information is not too explicit in the mirror geometry $\mathbf{W}$, since for example the symmetry between the three complex planes (and hence the difference among D7-branes wrapped on different 4-cycles $\left.z_{i}=0, i=1,2,3\right)$ seems to be realized simply by a shift in the elliptic fiber direction.
} 


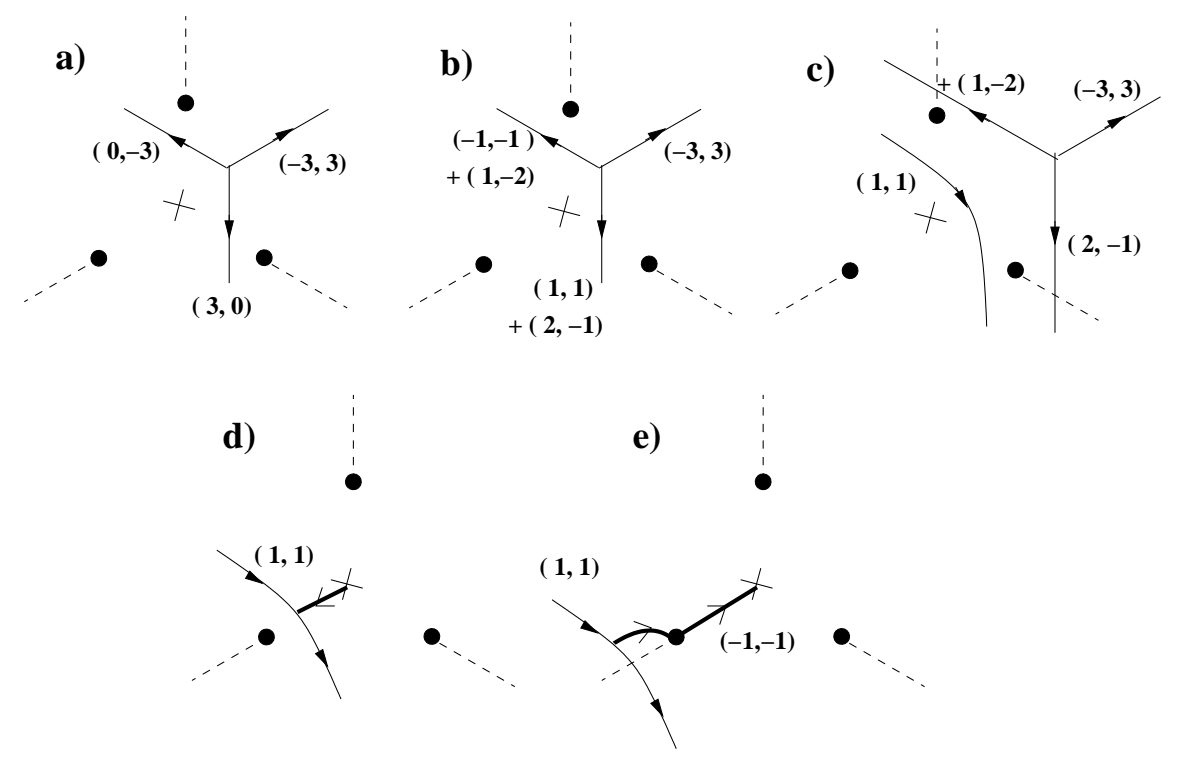

Figure 14: Geometric computation of the contribution of the non-compact 3-cycle $\Pi_{(-1,1)}$ to the compact homological charge, using by now familiar rules. The deformation argument leads to the relation $3\left[\Pi_{(-1,1)}\right]=\left[\Sigma_{(-1,-1)}\right]$.

Computing intersection number one obtains the generic structure of the resulting quiver diagram, which is shown in figure 15a. Using D6-branes wrapped on the basic compact 3-cycles and these non-compact 3-cycles it is straightforward to reproduce the models in Sections 3.3, 3.4 in [44]. One such example, with SM like spectrum is provided in figure $15 \mathrm{~b}$.

We would like however to emphasize that the original model, directly constructed in terms of 3-cycles in the threefold $\mathbf{W}$ (and without familiar mirror interpretation) is much simpler and phenomenologically more appealing (in particular, it avoids extra vector-like coloured triplets). This, in our view, shows that direct model building using local configurations of D6-branes on 3-cycles in non-compact threefolds (regardless of their mirror interpretation) is an interesting setup to explore new phenomenologically realistic D-brane constructions.

Nevertheless, we would like to conclude this section by remarking a fact: The realization of the same gauge sector within two completely different setups, namely Dbranes at singularities and intersecting D-branes, which are nevertheless related by a string duality (mirror symmetry). It is amusing to understand how both constructions manage to reproduce the same phenomenological properties (e.g. number of families arising from the structure of the singularity on $\mathbf{M}$ or from the intersection number 


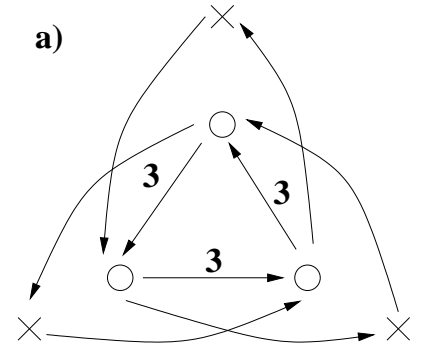

b)

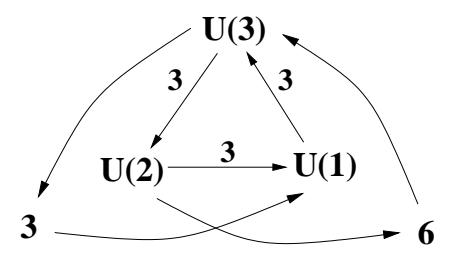

Figure 15: Figure a) shows the quiver diagram for the generic gauge theory sector on a set of D6-branes wrapped on 3-cycles providing the mirror of D3- and D7-branes at a $\mathbf{C}^{\mathbf{3}} / \mathbf{Z}_{\mathbf{3}}$ singularity. Figure b) shows the quiver diagram of a Standard Model like D6-brane configuration, mirror to a D3/D7 -brane configuration in 44 .

of 3-cycles on $\mathbf{W}$; etc). It is also interesting to understand they lead to the same phenomenological implications (e.g. gauge coupling unification at the point in moduli space with enhanced global $\mathbf{Z}_{\mathbf{3}}$ symmetry; superpotential couplings; etc). This fact has far reaching philosophical implications concerning our view on how string theory might reproduce the observed physics. Indeed, as emphasized in 45 the right question to ask is not which setup is the one realized in Nature (since all of them are related by string dualities, thus symmetries of Nature), but which setup provides the best description at the point of moduli space where moduli are eventually stabilized. For instance, the intersecting D-brane picture is most useful if moduli stabilize in a regime where 3-cycles have large sizes, while the picture of $\mathbf{D}$-branes at the $\mathbf{Z}_{\mathbf{3}}$ orbifold singularity may be most useful in the region near the CFT orbifold point (where the 3-cycles have stringy size and the intersecting brane picture is less useful).

It is easy to construct other examples of local configurations of wrapped D6-branes leading to interesting gauge field theories. Leaving a more detailed exploration for further work, we turn to discussing possible generalization of the above threefolds.

\section{Generalizations}

There are several simple generalizations of the above threefolds (2.1). We discuss them briefly in this section, leaving their more detailed exploration (which seems very promising) for future research. 


\subsection{Multiple $\mathrm{C}^{*}$ degenerations}

A simple generalization of the above ideas is to consider threefolds again given by a $\mathbf{T}^{\mathbf{2}} \times \mathbf{C}^{*}$ fibration over a complex plane, with $(p, q)$ degenerations of the elliptic fiber as above, but now with several points of degeneration of the $\mathbf{C}^{*}$ fibration. Such manifolds are described by

$$
\begin{aligned}
u v & =P(z)=\prod_{i=1}^{N}\left(z-z_{i}\right) \\
y^{2} & =x^{3}+f(z) x+g(z)
\end{aligned}
$$

Such manifolds are still Calabi-Yau 20. The rules to construct compact and noncompact 3-cycles are analogous to the above one, by fibering over networks of $(p, q)$ segments, which now are allowed to have ends on the diverse degeneration points of the $\mathbf{C}^{*}$ fibration. Intersection numbers between two such compact 3-cycles receive contributions only from intersection numbers of segments ending on the same $\mathbf{C}^{*}$ degeneration point. Intersection numbers of compact 3-cycles with 3-cycles spanning the non-compact direction in $\mathbf{C}^{*}$ may also receive contributions from intersections of segments away from the $\mathbf{C}^{*}$ degenerations. We skip the listing of detailed rules, since they should be clear from experience with previous threefolds.

Examples of such models are relatively similar to the constructions we have already considered, hence we turn to other possible generalizations.

\subsection{Double elliptic fibration}

Another possible generalization of the threefolds (2.1) is to consider a double elliptic fibration over the $z$ plane

$$
\begin{aligned}
& y^{2}=x^{3}+f(z) x+g(z) \\
& \tilde{y}^{2}=\tilde{x}^{3}+\tilde{f}(z) \tilde{x}+\tilde{g}(z)
\end{aligned}
$$

Namely the threefold is given by two different elliptic fibrations over the $z$-plane. At points in the $z$-plane, one of the two elliptic fibers $\mathbf{T}^{2}, \tilde{\mathbf{T}}^{2}$, degenerates due to the pinching of a $(p, q)$ or $(\tilde{p}, \tilde{q}) 1$-cycle. For suitable choices of elliptic fibrations the above non-compact threefold is Calabi-Yau. A global version of the above double elliptic fibration manifold has appeared in [47].

\footnotetext{
${ }^{20}$ In fact, can be regarded as blown-up versions of a $\mathbf{Z}_{\mathbf{N}}$ quotient of (2) by an order $N$ shift in the $\mathbf{S}^{\mathbf{1}}$ within the $\mathbf{C}^{*}$ fibration, $u \rightarrow e^{2 \pi i / N} u, v \rightarrow e^{-2 \pi i / N} v$, hence leaving the original point $u=v=z=0$ fixed.
} 
The basic compact 3-cycles in the manifold (3.2) are obtained by considering a segment stretching from a $(p, q)$ to a $(\tilde{p}, \tilde{q})$ degeneration points, and fibering over it the corresponding $(p, q)$ and $(\tilde{p}, \tilde{q})$ 1-cycles in the elliptic fibrations.

More generally, it is possible to consider 3-cycles arising from networks of segments in the $z$-plane, over which we fiber a two-cycle in the $\mathbf{T}^{\mathbf{2}} \times \mathbf{T}^{\mathbf{2}}$ fiber. One important subtlety is that the charge that should be conserved at junctions is the 2-homology class

$$
\begin{aligned}
{\left[\Pi_{2-\text { cycle }}\right] } & =(p[a]+q[b]) \otimes(\tilde{p}[\tilde{a}]+(\tilde{q}[\tilde{b}])= \\
& =p \tilde{p}[a] \otimes[\tilde{a}]+p \tilde{q}[a] \otimes[\tilde{b}]+q \tilde{p}[b] \otimes[\tilde{a}]+q \tilde{q}[b] \otimes[\tilde{b}]+
\end{aligned}
$$

Which in general is not addition of $(p, q)$ and $(\tilde{p}, \tilde{q})$ labels independently. Finally, the external legs in the network should have factorized 2-cycles fibered over them, and should end on the corresponding $(p, q)$ or $(\tilde{p}, \tilde{q})$ degenerations.

The intersection numbers between two 3-cycles are computed by careful addition with sign of the intersection numbers among the different segments. There are two contributions to the intersection numbers of segments: i) two segments with labels $(p, q),(\tilde{p}, \tilde{q})$ and $\left(p^{\prime}, q^{\prime}\right),(\tilde{p}, \tilde{q})$ ending on the same $(\tilde{p}, \tilde{q})$ degeneration contribute $I=$ $p q^{\prime}-q p^{\prime}$ to the intersection number (and similarly for exchanged roles of tilded and untilded); ii) an intersection in the $z$-plane of two segments with (in general nonfactorizable) 2-cycles $[\Pi],\left[\Pi^{\prime}\right]$, in the $\mathbf{T}^{\mathbf{2}} \times \mathbf{T}^{\mathbf{2}}$ fibered over them, contributes $I=[\Pi] \cdot\left[\Pi^{\prime}\right]$ to the intersection number (with - denoting intersection product in the $\mathbf{T}^{\mathbf{2}} \times \mathbf{T}^{\mathbf{2}}$ fiber).

Recalling that a $\mathbf{C}^{*}$ fibration near a degeneration point provides a local model for an elliptic fibration near a degeneration point, we notice that the geometries in section 3.1 provide a local model for double elliptic fibrations where all degenerations of one fibration are mutually local, namely of the same $(\tilde{p}, \tilde{q})$ type. In fact it is easy to recover the 3-cycles and their rules in section 3.1 by particularizing the ones in this section.

It is also a simple matter to generalize the discussion about supersymmetry in section 2.3 to the present context. For instance, a calibrating 3-form can be taken as

$$
\left[\Omega_{3}\right],=\left(d \tilde{x}_{1}+i d \tilde{x}_{2}\right) \wedge\left(d x_{1}+i d x_{2}\right) \wedge d z
$$

The basic 3-cycles we study are made of pieces of the form

$$
\begin{aligned}
x_{1}+i x_{2} & =p+\tau q=a e^{i \theta_{1}} \\
\tilde{x}_{1}+i \tilde{x}_{2} & =\tilde{p}+\tilde{\tau} \tilde{q}=b e^{i \theta_{2}} \\
z & =c e^{i \theta_{3}}
\end{aligned}
$$



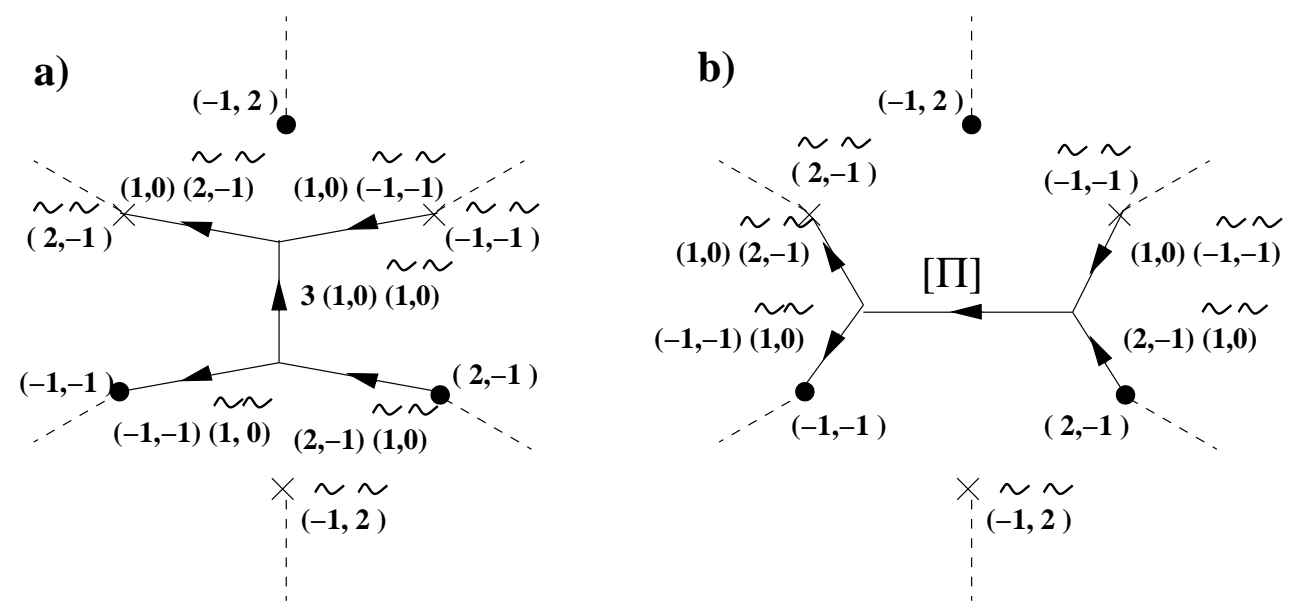

Figure 16: Figure a) shows a 3-cycle in a double elliptic fibration threefold. Note the conservation of 2-homology charge in the $\mathbf{T}^{\mathbf{2}} \times \mathbf{T}^{\mathbf{2}}$ fiber as one moves across junctions. Figure b) shows another representative 3-cycle in the same homology class, which is slag in the $\mathbf{Z}_{\mathbf{3}}$ symmetric configuration. Notice that one of the segments has a non-factorizable 2-cycle (in the class $\left.[\Pi]=\left[a_{1}\right]\left[a_{2}\right]-\left[a_{1}\right]\left[b_{2}\right]-\left[a_{2}\right]\left[b_{1}\right]\right)$ in the $\mathbf{T}^{\mathbf{2}} \times \mathbf{T}^{\mathbf{2}}$ fiber.

parametrized by $a, b, c \in \mathbf{R}$, so that the cycle is slag if each segment satisfies $\theta_{1}+\theta_{2}+$ $\theta_{3}=0$.

Notice that at some junctions, factorizable 2-cycles in the double elliptic fiber may combine into a non-factorizable 2-cycle. We expect that the latter will lead to a slag segment if the former do. A particular example of this is given below (figure 16b).

We would like to conclude by giving a simple example of a D6-brane configuration in such a double elliptic fibration. Consider each double elliptic fibration to have the following set of degenerations: $(2,-1),(-1,2),(-1,-1)$, which we order in a counterclockwise fashion as in figure 16 (this implies a choice of point in the moduli space). This space is a Calabi-Yau threefold. Notice that at this point in moduli space there is a geometric $\mathbf{Z}_{\mathbf{3}}$ symmetry similar to (2.24), with the monodromy matrix now acting on both elliptic fibers.

In Figure 16a) we present one particular compact 3-cycle in this threefold. Notice that the 2-homology charge in the $\mathbf{T}^{\mathbf{2}} \times \mathbf{T}^{\mathbf{2}}$ fiber is conserved across junctions. At the $\mathbf{Z}_{\mathbf{3}}$ symmetric configuration, the 3-cycle in figure 16a) has a slag representative shown in figure 16b). Two other compact 3-cyles may be obtained by acting with the $\mathbf{Z}_{\mathbf{3}}$ geometrical symmetry.

Let us consider a set of $N$ D6-branes wrapped on each of these three compact 
3-cycles. It is possible to check that the resulting configuration cancels all localized RR tadpoles 21. Finally by computing intersecting numbers, the final four-dimensional chiral gauge theory has the quiver in figure $7 \mathrm{~b}$. Hence our present example gives a different construction of the chiral gauge field theory in section 2.5.1. It is then clear that double elliptic fibrations have enough richness to yield interesting local configurations of wrapped D6-branes.

\subsection{Higher genus fibrations}

There is a final class of generalization that we would like to mention. These new geometries are obtained by fibering over a complex plane a $\mathbf{C}^{*}$ fibration and a fibration of a higher genus curve $\Sigma_{g}$. Over certain points in the base $z$-plane a 1-cycle of the genus $g$ curve fiber degenerates, hence degeneration points are labeled by the 1-homology class of the degenerating 1-cycle $[\Pi]=\sum_{r=1}^{g}\left(p_{r}\left[a_{r}\right]+q_{r}\left[b_{r}\right]\right)$.

In analogy with previous examples, one can construct a set of compact 3-cycles by taking segments in the $z$-plane stretching between the degeneration point of the $\mathbf{C}^{*}$ fibration and one of the degeneration points of the $\Sigma_{g}$ fibration, and fibering over it the $\mathbf{S}^{\mathbf{1}}$ in the $\mathbf{C}^{*}$ fiber and the corresponding 1-cycle [ח] in $\Sigma_{g}$. More generally, one can construct more complicated 3-cycles by considering networks of segments in the $z$-plane, over which one fibers the $\mathbf{S}^{\mathbf{1}}$ in the $\mathbf{C}^{*}$ fiber and a 1 -cycle $[\Pi]$ in $\Sigma_{g}$, and ensuring conservation of the 1-homology class in $\Sigma_{g}$ across junctions in the network.

The intersection number between two such 3-cycles is given by adding contributions from segment intersection numbers. The latter only receive contributions from two segments, of $[\Pi],\left[\Pi^{\prime}\right]$ kinds, ending on the $\mathbf{C}^{*}$ degeneration point. The contribution is given by

$$
[\Pi] \cdot\left[\Pi^{\prime}\right]=(\vec{p} ; \vec{q}) \cdot \mathbf{I} \cdot\left(\vec{p}^{\prime} ; \vec{q}^{\prime}\right)=\vec{p} \cdot \vec{q}^{\prime}-\vec{q} \cdot \vec{p}^{\prime}
$$

where $(\vec{p} ; \vec{q})=\left(p_{r} ; q_{r}\right)$ and $\mathbf{I}=\left(\begin{array}{cc}0 & \mathbf{1}_{g} \\ -\mathbf{1}_{g} & 0\end{array}\right)$ is the intersection form in $\Sigma_{g}$.

A few particular examples of Calabi-Yau manifolds of this kind have been considered in the literature 48, 17]. Their introduction is motivated from consideration of the manifolds mirror to orbifold singularities $\mathbf{C}^{\mathbf{3}} / \mathbf{Z}_{\mathbf{N}}$, other than $\mathbf{Z}_{\mathbf{3}}$. Indeed, the particular

\footnotetext{
${ }^{21} \mathrm{~A}$ simple proof is to start with a homologically trivial small loop at the center of the configuration, deform it to grow prongs to and from the elliptic degenerations, shrink the initial small loop, and then suitably recombine the prongs to reproduce the three 3-cycles. By similar arguments, the general RR tadpole condition for this threefold can be shown to be that the net number of outgoing prong from the $i^{\text {th }}$ elliptic degeneration should be $i$-independent.
} 
a)

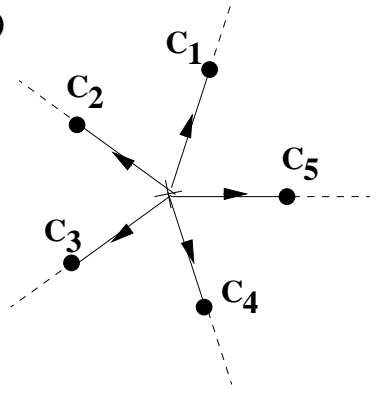

b)

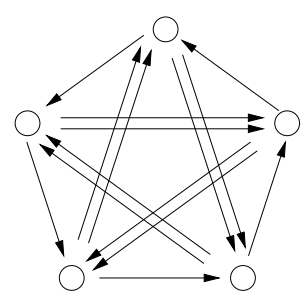

Figure 17: Figure a) shows a configuration of D6-branes wrapped on the basic compact 3 -cycles in a Calabi-Yau threefold given by a $\mathbf{C}^{*}$-fibration and a genus 2 curve fibration over the complex plane. Figure b) gives the quiver associated to the corresponding chiral gauge field theory.

case of the mirror of the $\mathbf{Z}_{\mathbf{5}}$ geometry was obtained from the mirror map in [48], and is given by a genus 2 curve fibration with a set of five degenerate fibers, with labels

$$
\begin{gathered}
C_{1}=(0,0 ;-1,2) \quad ; \quad C_{2}=(-1,0 ; 0,-3) \quad ; \quad C_{3}=(0,1 ; 4,-1) \\
C_{4}=(0,-1 ;-1,0) \quad ; \quad C_{5}=(1,0 ;-2,2)
\end{gathered}
$$

The resulting set of basic compact 3-cycles and the quiver for the chiral gauge theory obtained upon wrapping D6-branes on them are shown in figure 17. Notice that it reproduces the gauge theory on a regular D3-brane at the $\mathbf{C}^{\mathbf{3}} / \mathbf{Z}_{\mathbf{5}}$ singularity, a system related to ours by mirror symmetry.

Certain features of other examples, as well as a discussion of the different compact 3 -cycles in such geometries, have appeared in [17].

Even though the explicit example above was provided by mirror symmetry, it is clear that given a threefold of the kind described above one may use D6-branes wrapped on different sets of 3-cycles to generate other chiral gauge sectors. However, the constructions of such manifolds and cycles is relatively technical and lies beyond the scope of the present paper. Clearly, further generalizations by introducing further degenerations of the $\mathbf{C}^{*}$ fibration, or extending it to a new elliptic fibration should be clearly tractable from the constructions above.

\subsection{Orientifold models}

It is straightforward to inmplement orientifold projections in the threefolds in section 2 or their generalizations, whenever they have a geometric $\mathbf{Z}_{\mathbf{2}}$ symmetry. We will not 
explore the different possibilities of model building in detail, but we expect them to be very rich, and very likely to yield the Standard Model intersection numbers of [3] in suitable examples.

Any threefold with a $\mathbf{Z}_{\mathbf{2}}$ geometric symmetry $g$ may be orientifolded by quotienting the configuration by $\Omega g$ (times $(-1)^{F_{L}}$ if required). The configuration of wrapped D6branes should be $\mathbf{Z}_{\mathbf{2}}$ invariant, via the introduction of orientifold images, and should satisfy RR tadpole cancelation conditions that now may include the contribution from the orientifold plane charges.

We would like to point out that, if one is not interested in supersymmetric configurations, the non-compact setup allows in principle to construct models with orientifold planes which are not O6-planes, since the corresponding RR charges can escape to infinity. This possibility was not available in the compact setup, so the present framework may allow for more freedom 22.

There exist examples of such $\mathbf{Z}_{\mathbf{2}}$ symmetries in particular examples of threefolds. To give an example, if all the elliptic fibration degenerations are mutually local (say all of $(1,0)$ type), and are distributed $\mathbf{Z}_{\mathbf{2}}$ symmetrically with respect to the origin in the $z$-plane, we may mod out by $\Omega$ times

$$
z \rightarrow-z \quad ; \quad x_{1} \rightarrow x_{1} \quad ; \quad x_{2} \rightarrow-x_{2} \quad ; \quad u \rightarrow-u \quad ; \quad v \rightarrow-v
$$

The action on $x_{1}+i x_{2}$, the complex coordinate on the elliptic fiber, is equivalent to $y \rightarrow \bar{y}, x \rightarrow \bar{x}$ in (2.1). The orientifold planes span a complex curve in $u, v, z$ times a real line in $x_{1}+i x_{2}$, so the model contains O4-planes. Their RR flux may escape to infinity, so there are no tadpoles cancellation condicions associated to them. Other special examples of $\mathbf{Z}_{\mathbf{2}}$ symmetries in particular threefolds are easily constructed and may be useful in model building.

Clearly there are many possibilities. This kind of projections would break the supersymmetries of the configuration, but many models in the literature are already non-supersymmetric (in the spirit of [49]). Another interesting fact is that most of these orientifold will not contribute to the tadpoles for the RR fields arising from integrals of the RR 7-form over compact 3-cycles, hence they do not modify the RR tadpole conditions for D6-branes. Indirectly (via the relation between tadpoles and anomalies) this suggests that the matter content on the gauge theory may be free of

\footnotetext{
${ }^{22}$ On the other hand, one should note that if the O-planes introduced span too many non-compact directions they may lead to other localized RR tadpoles requiring its own set of D-branes. However the latter typically would not lead to chiral matter on the D6-brane gauge theory, so the net effect is similar to simply forgetting their tadpoles.
} 
two-index tensor representations of the gauge groups (or may less fortunately lead to equal number of symmetric and antisymmetric representations). This is important for phenomenological purposes, since such fields tend to yield exotics not present in the Standard Model (see e.g. [6]).

There are also the more familiar orientifold projections yielding O6-planes wrapped on 3-cycles of the kind studied above. These are obtained when the geometric $\mathbf{Z}_{\mathbf{2}}$ acts antiholomorphically on the complex coordinates of the threefold. It is straighforward to find examples of such actions.

For instance, starting with (2.1) with elliptic degenerations $(2,-1),(-1,2),(-1,-1)$ in the $\mathbf{Z}_{\mathbf{3}}$ symmetric configuration, we may mod by

$$
u \rightarrow \bar{v} \quad ; \quad v \rightarrow \bar{u} \quad ; \quad z \rightarrow \bar{z} \quad ; \quad x_{1} \rightarrow-x_{1} \quad ; \quad x_{2} \rightarrow x_{2}
$$

where $x_{1}+i x_{2}$ is the complex coordinates in the $\mathbf{T}^{\mathbf{2}}$ fiber. The O6-plane spans the imaginary axis in the $z$-plane, the $\mathbf{S}^{\mathbf{1}}$ in $\mathbf{C}^{*}$ and the 1-cycle $(1,-2)$ in the elliptic fiber. Hence it contributes an amount of $-4\left[\Sigma_{(1,-2)}\right]$ to the localized RR tadpole, with the -4 arising from the O6-plane charge.

Let us build a configuration of wrapped D6-branes in this orientifolded threefold background. Wrapping $N$ D6-branes on the invariant 3-cycle $\left[\Sigma_{(-1,2)}\right]$, and $N+4$ D6branes on the $\left(\mathbf{Z}_{\mathbf{2}}\right.$-related) 3-cycles $\left[\Sigma_{(2,-1)}\right]$, $\left[\Sigma_{(-1,-1)}\right]$, the configuration is tadpole-free. The gauge theory spectrum is

$$
\begin{array}{rr}
\text { Gauge group } & S O(N) \times U(N+4) \\
\mathcal{N}=1 \text { Ch. Mult. } & 3(\square, \bar{\square}, 1)+3(1, \boxminus)
\end{array}
$$

which can be codified in an orientifolded quiver [41]. Choosing $N=1$, we recover a three-family $S U(5)$ grand unified model. In fact, this is nothing but the mirror of a (local version of a) model in [50].

Hoping that these examples suffice to observe the general rules in orientifold constructions, we leave the detailed exploration of other explicit examples for future research. See one further example in section 4 .

\section{Local intersecting brane-worlds in a two-torus times a non-compact two-fold}

In this section we describe the construction of yet another class of non-compact threefolds. They are simpler than the above ones, yet we have preferred to postpone their 
discussion since in a sense they have a less general structure.

The manifolds we would like to consider are a global product of a two-torus times a non-compact two-fold $\mathbf{X}$. In principle we would like to restrict to the Calabi-Yau situation, which enforces $\mathbf{X}$ to be either flat or a piece of a K3. For convenience, and also because it parallels previous analysis, we choose to describe our two-fold as an elliptic fibration over a complex plane.

$$
y^{2}=x^{3}+f(z) x+g(z)
$$

Note that the geometry is very similar to the double elliptic fibrations in section 3.2, with one elliptic fibration being trivial, i.e. without degeneration points. Hence the rules below are easily obtained by particularizing those in section 3.2 .

On this geometry we can consider different kinds of 3-cycles. The basic ones can be constructed by taking the product of a 1-cycle $(m, n)$ in the two-torus, times a segment in the $z$-plane, over which we fiber a 1-cycle $(p, q)$ in the elliptic fibration. We will describe this pictorially by a segment in the $z$-plane carrying two labels $(m, n)$ and $(p, q)$. For the 3 -cycle to be closed, we need both ends of the segment to end on $(p, q)$ degenerations of the elliptic fibration.

A slight generalization is to consider segments over which we fiber a two-cycle in the homology class $[\Pi]$ in $\mathbf{T}^{\mathbf{2}} \times \mathbf{T}^{\mathbf{2}}$, where the last factor is the elliptic fiber. For non-factorizable $[\Pi]$ such 3 -cycles are non-compact, since they are unable to end on degeneration points of the elliptic fibration.

As usual, we may also consider more complicated 3-cycles, obtained by considering a network of $[\Pi]$ segments over the $z$-plane, over which we fiber the corresponding 2-cycles in $\mathbf{T}^{\mathbf{2}} \times \mathbf{T}^{\mathbf{2}}$, and ensuring conservation of the 2-homology class $[\Pi]$ across junctions. In order to obtain compact 3-cycles, the external legs in the network must have factorizable $[\Pi]$ and must end on the corresponding $(p, q)$ degeneration of the elliptic fibration.

The intersection number of two 3-cycles receives two kinds of contributions from segment intersection numbers: i) two segments with labels $(m, n),(p, q)$, and $\left(m^{\prime}, n^{\prime}\right)$, $(p, q)$ ending on the same $(\tilde{p}, \tilde{q})$ degeneration contribute $I=m n^{\prime}-n m^{\prime}$ to the intersection number; ii) an intersection in the $z$-plane of two segments with (in general non-factorizable) labels $[\Pi],\left[\Pi^{\prime}\right]$, contributes $I=[\Pi] \cdot\left[\Pi^{\prime}\right]$ to the intersection number (with $\cdot$ denoting intersection product in $\mathbf{T}^{\mathbf{2}} \times \mathbf{T}^{\mathbf{2}}$ ).

Notice that factorizable 3-cycles contain at least one non-trivial element in its first homology group (since they are a product of $\mathbf{S}^{\mathbf{1}}$ in $\mathbf{T}^{\mathbf{2}}$ times a 2-cycle in $\mathbf{X}$ ). Hence, D6-branes on such 3-cycles lead to at least one adjoint chiral multiplet. 
For simplicity, below we consider some examples of this kind of construction for a particularly simple kind of elliptic fibration, where all degenerations are of the same $(p, q)$ kind, which without loss of generality we take to be $(1,0)$. Other case may be analyzed using the above rules.

An interesting feature of the elliptic fibration with $N(1,0)$ degenerations is that it provides the mirror manifold of a $\mathbf{C}^{2} / \mathbf{Z}_{\mathbf{N}}$ ALE singularity 23 . Hence the models with D6-branes wrapped on compact 3-cycles may be related via two-fold mirror symmetry with models of D-branes wrapped on 1-cycles in $\mathbf{T}^{\mathbf{2}}$ and on holomorphic cycles in the $\mathbf{C}^{\mathbf{2}} / \mathbf{Z}_{\mathbf{N}}$ singularity. In particular cases, such B-type branes can be described as D-branes sitting at the singular point in $\mathbf{C}^{\mathbf{2}} / \mathbf{Z}_{\mathbf{N}}$. Hence the models of D6-branes on compact 3-cycles in the threefold may be in some cases related to models of D4-branes wrapped on 1-cycles on $\mathbf{T}^{\mathbf{2}}$ and sitting at a $\mathbf{C}^{\mathbf{2}} / \mathbf{Z}_{\mathbf{N}}$ singularity. The latter models were introduced in [2] (see also [51, 52]). In this sense, our construction in this section provides a generalization of such models, including configurations not related to D4brane models. In particular we would expect that the general case of D6-branes on 3-cycles on $\mathbf{T}^{\mathbf{2}} \times \mathbf{X}$ to avoid certain unwanted features of D4-branes models, like the generic presence of tachyons at intersections.

In figure 18a we show a particular choice of D6-branes wrapped on compact 3cycles in $\mathbf{T}^{\mathbf{2}}$ times an elliptic fibration with three $(1,0)$ degenerations. Cancellation of localized RR tadpoles is easily checked by a deformation argument. The spectrum of the resulting chiral gauge theory is shown in the quiver diagram of figure 18b. The configuration precisely reproduces the D4-brane model in section 4.3 in the first reference in [2], to which it is related via mirror symmetry in $\mathbf{X}$. Notice also that certain features like the generic presence of tachyons can be tracked to the impossibility of making the different overlapping 3-cycles simultaneously slag, no matter the location of the elliptic fibration degenerations (i.e. no matter at which point in moduli space one is sitting).

Notice that the spectrum is quite close to that of the Standard Model. An important issue is to obtain a massless hypercharge. As in [2] one could in principle claim that there is an anomaly free linear combination of $U(1)$ 's which yields correct hypercharge asignments. However, such combination does not pass the test (subsequently brought to attention in [3], but still too often overlooked in the literature) of having no $B \wedge F$ couplings with four-dimensional 2-form fields. Hence the model has no hypercharge

\footnotetext{
${ }^{23}$ In fact such singularities are self-mirror, so it is also true that the fibration $\mathbf{X}$ is a blown-up version of $\mathbf{C}^{\mathbf{2}} / \mathbf{Z}_{\mathbf{N}}$. We prefer to regard it as its mirror to make it consistent with the mapping of branes mentioned below.
} 
a)

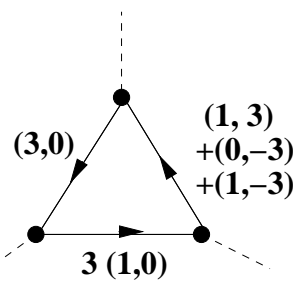

b)

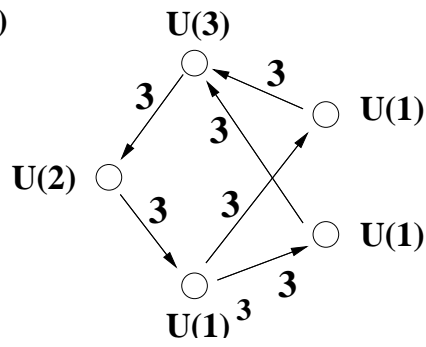

Figure 18: Local configuration of D6-branes wrapped on compact 3-cycles in a non-compact manifold $\mathbf{T}^{\mathbf{2}} \times \mathbf{X}$. The 3 -cycles are products of 1 -cycles $(m, n)$ in $\mathbf{T}^{\mathbf{2}}$ (these are the labels in the figure) times 2-cycles in $\mathbf{X}$. The latter are obtained by fibering over each segment the $(1,0)$ 1-cycles in the elliptic fibration, since this is the only kind of degeneration. Notice the change of notation with respect to other figures, namely the $(m, n)$ labels on the segments refer to the 1-cycle in $\mathbf{T}^{\mathbf{2}}$ rather than to on the elliptic fiber.

a)

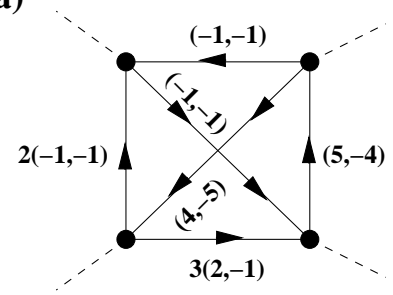

b)

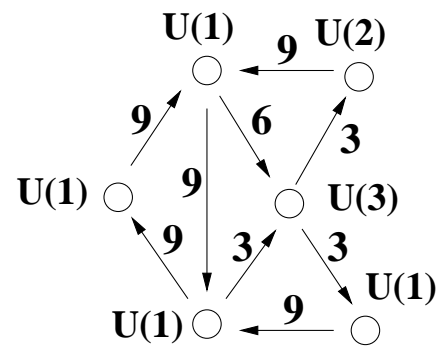

Figure 19: Yet another example of configurations of D6-branes on a threefold $\mathbf{T}^{\mathbf{2}} \times \mathbf{X}$. As in figure 18, labels in figure a) refer to the $(m, n)$ charge in $\mathbf{T}^{\mathbf{2}}$, whereas the $(p, q)$ charge in the elliptic fiber is $(1,0)$ for all cycles. Figure b) shows the quiver diagram for the resulting gauge theory.

candidate and should be regarded as a simple toy model for our construction. This seems to be a general feature of models of D6-branes wrapped on only compact 3cycles in $\mathbf{T}^{\mathbf{2}} \times \mathbf{X}$. Introduction of non-compact 3-cycles presumably allows to avoid such problems in more involved models.

In figure 19 we present another example of wrapped D6-brane configuration, which is not mirror of any configuration of D4-branes. It yields a gauge theory Standard Model like group and three chiral families, but unfortunately also no hypercharge candidate (and plenty of extra matter). It should not be difficult to modify examples of this kind e.g. by introducing non-compact D6-branes and build phenomenologically 

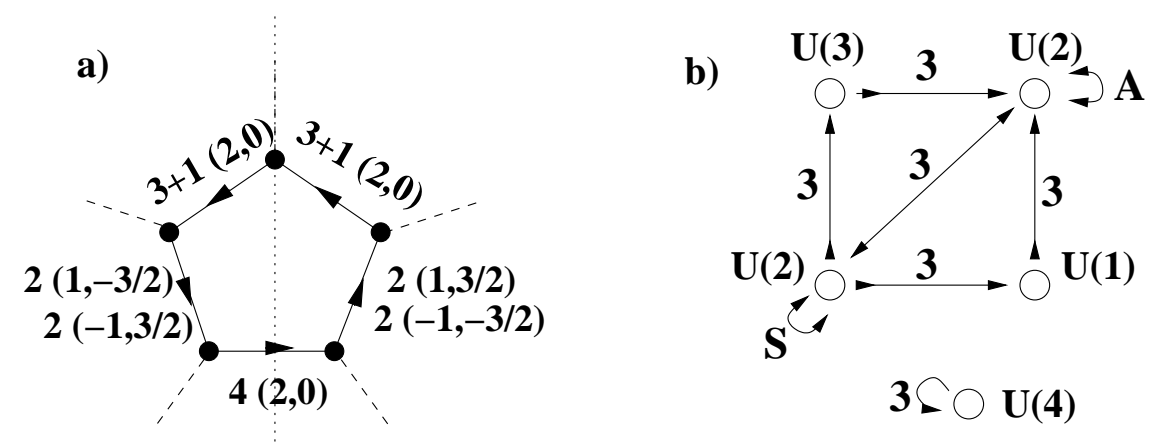

Figure 20: A simple example of D6-branes wrapped on 3-cycles in an orientifold of the $\mathbf{T}^{\mathbf{2}} \times \mathbf{X}$ geometry, along with its quiver diagram. Conventions are as in other figures in this section. Note that quiver arrows may have two outgoing ends in an orientifold model 441. Also, $\mathbf{S}$ and $\mathbf{A}$ denote two index symmetric and antisymmetric representations.

more appealing models.

Clearly it is possible to implement orientifold projections in the present setup. One of the simplest possibilities is to consider only $(1,0)$ degenerations of the elliptic fibration, and distribute them in a $\mathbf{Z}_{\mathbf{2}}$ invariant fashion in the $z$-plane (say, $z \rightarrow-\bar{z}$ ). One may then mod out by $\Omega$ times

$$
z \rightarrow-\bar{z} \quad ; \quad x_{1} \rightarrow x_{1} \quad ; \quad x_{2} \rightarrow-x_{2} \quad ; \quad u \rightarrow-\bar{v} \quad ; \quad v \rightarrow \bar{u}
$$

This introduces O6-planes wrapped on the $\mathbf{S}^{\mathbf{1}}$ in $\mathbf{C}^{*}$, the imaginary axis in the $z$ plane, and the $(1,0)$ 1-cycle in the elliptic fiber. The contribution of the O6-plane to the RR tadpole cancellation is computable from deformation arguments, or checking intersection numbers. Such orientifold geometries are typically mirror of orientifolds of $\mathbf{T}^{\mathbf{2}} \times \mathbf{C}^{\mathbf{2}} / \mathbf{Z}_{\mathbf{N}}$ orbifolds. Inclusion of wrapped D6-branes (and their $\mathbf{Z}_{\mathbf{2}}$ images) lead to intersecting brane worlds, which may have a simple mirror or not (as models of D4-branes at the orbifold geometry), depending on the kind of wrapped branes used. Rules to compute the spectrum follow from standard arguments and it would be lengthy to attempt a general list here. Let us instead present an illustrative exaple of a simple tadpole free configuration is given in figure 20. This particular example contains branes with simple mirror D4-brane interpretation; indeed the model is mirror to the D4-brane model in appendix I in [51]. It corresponds to a left-right symmetric model $S U(3) \times S U(2)_{L} \times S U(2)_{R} \times U(1)_{B-L}$ with three families of quarks and leptons. 


\section{Configurations with Standard Model intersec- tion numbers}

\subsection{Construction of the configurations}

In this Section we would like to address a last issue. In [3] a set of intersection numbers for 3-cycles in orientifolded Calabi-Yau models were proposed, such that, when wrapped by D6-branes, lead to a gauge sector with exactly the chiral fermion content of the standard model. Particular realizations of 3-cycles with those intersection numbers has been achieved in orientifolds of the six-torus [3], certain orbifolds [51] and the quintic Calabi-Yau threefold [7].

It would be extremely interesting to realize such intersection numbers in noncompact threefolds of the kind studied in this paper. A superficial direct search has not led to this structure, but we certainly do expect such models to exist, and be classifiable once more formal exploration tools are developed. The threefolds we have studied seem rich enough to allow intersection patters as specific as those in [3]. We expect much progress in this direction.

In this Section we introduce a new class of non-compact threefolds, which are not Calabi-Yau, but have the interesting property of leading, in a relatively simple way to the Standard Model intersection numbers in [0]]. The threefolds we consider are topologically $\mathbf{T}^{\mathbf{2}} \times \mathbf{T}^{\mathbf{2}} \times \mathbf{Y}$, with $\mathbf{Y}$ a non-compact Riemann surface. The motivation to use these threefold will become clear below. Since the geometry of the onefold $\mathbf{Y}$ and its set of compact 1-cycles are quite different from previous geometries, we briefly describe them.

Any non-compact Riemann surface can be conveniently described as a double cover of the complex $z$-plane, with a certain number $N$ of branch points $z_{i}$

$$
y^{2}=\prod_{i=1}^{N}\left(z-z_{i}\right)
$$

Branch cuts connecting the two sheets of the cover emanate from the branch points, and end on other branch cuts or go off to infinity. The double cover representation of the 2-torus (minus one point to make it non-compact) is shown in figure 21.

In this geometry, non-trivial 1-cycles are described by closed paths surrounding two branch points. If both share a common branch cut, the 1-cycle lives in one of the sheets; whereas if the two branch points correspond to different branch cuts the resulting 1-cycle crosses them and contains pieces in both sheets. Other 1-cycles may 


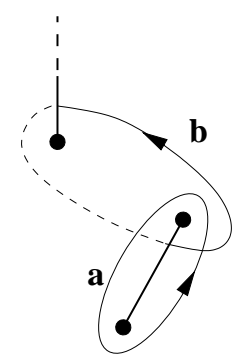

Figure 21: Double cover representation of the two-torus (minus one point). Thick lines correspond to branch cuts joining the two sheets. Thin lines show the two non-trivial $a$ and $b$ 1-cycles; continuous and dashed pieces live in different sheets of the cover.

be constructed by combining the above basic set. Let us note for completeness that the 1-cycles can be deformed, preserving the homology class, keeping in mind to change the sheet whenever they cross a branch cut. Examples of the non-trivial $a$ and $b 1$-cycles in the 2-torus are shown in figure 21. Hence the number of branch points determines the number of holes in the Riemann surface, whereas the number of boundary components is determined by the structure of branch cuts going off to infinity. For simplicity we consider double covers where the number of branch cuts going to infinity is minimal, namely zero or one for even or odd $N$ (other cases are similar). We also choose a canonical representation, to be used in topological matters, where branch points are counterclockwise ordered in a circle in the $z$-plane, with branch cuts starting at $z_{2 k-1}$ and ending at $z_{2 k}$ (and a last branch cut going off to infinity outwards from the unpaired branch point for odd $N$ ).

An interesting property to be used below is that there exists a simple set of 1cycles, denoted $\gamma_{(i, i+1)}$, defined as closed paths surrounding (counterclockwise) the branch points $z_{i}, z_{i+1}$, and whose only non-vanishing intersection numbers are

$$
\gamma_{(i-1, i)} \cdot \gamma_{(i, i+1)}=1
$$

with $i$ defined modulo $N$. Moreover, it is straightforward to use a deformation argument to show the homological relation

$$
\sum_{i=1}^{N}\left[\gamma_{(i, i+1)}\right]=0
$$

Examples of this set for even and odd $N$ are shown in figure 22 .

We are interested in configurations of D6-branes wrapped on 3-cycles in $\mathbf{T}^{\mathbf{2}} \times \mathbf{T}^{\mathbf{2}} \times \mathbf{Y}$, given by a product of three 1-cycles, one per factor (As usual we skip the discussion of 
a)

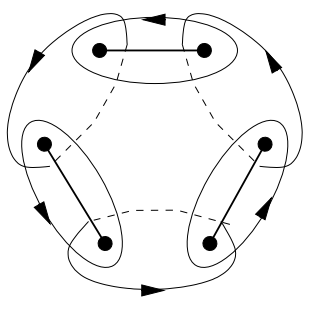

b)

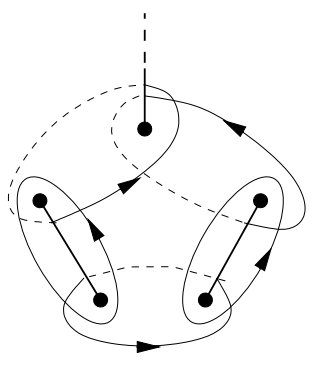

Figure 22: A set of non-trivial $\gamma_{(i, i+1)}$ 1-cycles in two Riemann surfaces with even and odd $N$.

non-factorizable 3-cycles). Using 1-cycles of $\mathbf{Y}$ of the kind described above, each stack of $\mathrm{D} 6_{a}$-branes is labeled by its wrapping numbers in $\mathbf{T}^{\mathbf{2}} \times \mathbf{T}^{\mathbf{2}}$, namely $\left(n_{a}^{1}, m_{a}^{1}\right),\left(n_{a}^{2}, m_{a}^{2}\right)$

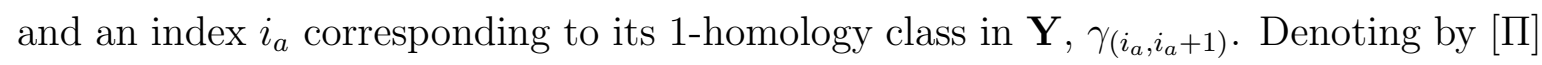
the 2-homology class of the 3-cycle in $\mathbf{T}^{\mathbf{2}} \times \mathbf{T}^{\mathbf{2}}$, the intersection number between the 3-cycles associated to the $a^{\text {th }}$ and $b^{\text {th }}$ stack is

$$
I_{a, b}=\left[\Pi_{a}\right] \cdot\left[\Pi_{b}\right] \times\left(\delta_{i_{b}, i_{a}+1}-\delta_{i_{b}, i_{a}+1}\right)
$$

This number determines the number of arrows joining the nodes associated to $a$ and $b$ in the quiver diagram.

The contributions to the RR tadpoles are easily computed by checking intersection numbers in the generic quiver, or by using homological relations like (5.3) from deformation arguments. For instance, denoting $\left[\Pi_{i}\right]$ the total 2-homology class in $\mathbf{T}^{\mathbf{2}} \times \mathbf{T}^{\mathbf{2}}$ associated with D6-branes on wrapped on the 1-cycle $\gamma_{(i, i+1)}$ in $\mathbf{Y}$, we may guarantee cancellation of $\mathrm{RR}$ tadpoles by requiring

$$
\sum_{i=1}^{N}\left[\Pi_{i}\right]=0
$$

Instead of building examples of this kind, let us introduce a further ingredient required to reproduce the Standard Model instersection numbers, namely the orientifold projection. We may for instance mod out by $\Omega$ times

$$
z_{1} \rightarrow \bar{z}_{1} \quad ; \quad z_{2} \rightarrow \bar{z}_{2} \quad ; \quad z \rightarrow \bar{z}
$$

on the complex coordinates $z_{1}, z_{2}$ of $\mathbf{T}^{\mathbf{2}} \times \mathbf{T}^{\mathbf{2}}$, and on (5.1). Such symmetry is present for onefolds $\mathbf{Y}$ with a set of branch points and cuts invariant with respect to the imaginary $z$ axis, as in figure 22. Also the 2-tori are restricted to be rectangular 
or tilted at a particular angle. In the latter case, we introduce (possibly fractional) effective wrapping numbers $(n, m)$ with $m$ integer or half-odd if $n$ is even or odd [4].

We would like to describe an orientifold action on the homology classes of 3-cycle. Consider for concreteness the case of odd $N$, where due to $\mathbf{Z}_{\mathbf{2}}$ symmetry the unpaired branch cut must lie at imaginary $z$. Labelling such branch point as $i=(N+1) / 2$, the orientifold action on 3-cycles we are interested in is to change their labels as follows 24

$$
\left(n^{1}, m^{1}\right),\left(n^{2}, m^{2}\right), i \rightarrow\left(n^{1},-m^{1}\right),\left(n^{2},-m^{2}\right),-i
$$

The contribution of the O6-plane to RR tadpoles can be computed using intersection numbers or deformation arguments. With this information it is now possible to look for configurations of D6-branes on 3-cycles which reproduce the Standard Model intersection numbers. However, instead of performing a brute force attempt, it will be useful to note that the same algebraic problem has already appeared in a different (but possibly related, see below) context. Such small detour will also make manifest our motivation to study the above threefolds and the above set of 1-cycles in looking for the Standard Model intersection numbers.

One particular realization of the spectrum of just the Standard Model has been provided in [51] in (orientifolded) configurations of D5-branes wrapped on 2-cycles on $\mathbf{T}^{\mathbf{2}} \times \mathbf{T}^{\mathbf{2}}$ and sitting at a $\mathbf{C} / \mathbf{Z}_{\mathbf{N}}$ singularity. In such models, stacks of D5-branes are labeled by an index $i$ defined modulo $N$, defining its Chan-Paton eigenvalue with respect to the $\mathbf{Z}_{\mathbf{N}}$ action. The chiral spectrum from strings stretched between the $a^{\text {th }}$ and $b^{\text {th }}$ stacks is given by

$$
I_{a, b}=\left[\Pi_{a}\right] \cdot\left[\Pi_{b}\right] \times\left(\delta_{i_{b}, i_{a}+1}-\delta_{i_{b}, i_{a}+1}\right)
$$

where the last piece arises from the quiver structure of the $\mathbf{C} / \mathbf{Z}_{\mathbf{N}}$ singularity.

The key point is that the numbers of bi-fundamental chiral fermions (5.8) in D5brane models exactly coincides with the intersection numbers of 3-cycles in $\mathbf{T}^{\mathbf{2}} \times \mathbf{T}^{\mathbf{2}} \times \mathbf{Y}$. Moreover, the orientifold action on D5-brane stacks exactly coincides with the label exchange (5.7). Similarly, the translation of constraints (5.5) to D5-brane language reproduces the cancellation of twisted $R R$ tadpoles for D5-branes at $\mathbf{C} / \mathbf{Z}_{\mathbf{N}}$.

\footnotetext{
${ }^{24}$ Strictly speaking the orientifold action 5.6 does not act like this on 1-cycles in $\mathbf{Y}$. On the other hand, it does act in this fashion on certain linear combinations of 1-cycles, which have the same intersection as the original ones. This implies that there exists an orientifold action, related to (5.6) by a change of variables (symplectic transformation), which acts on 1-cycles as (5.7). To simplify the discussion we stick to the latter.
} 
a)

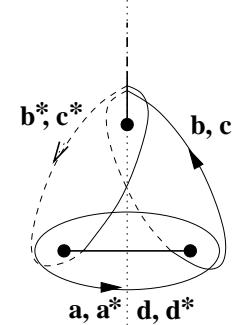

b)

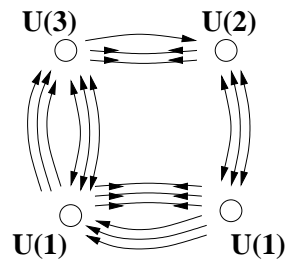

Figure 23: Figure a) shows the structure of cycles used in the construction of the Standard Model example. Figure b) provides the quiver diagram for the resulting gauge theory, which indeed reproduces the Standard Model (plus right-handed neutrinos).

Hence the problem of finding explicit realizations of configurations of D6-branes wrapped on 3-cycles in $\mathbf{T}^{\mathbf{2}} \times \mathbf{T}^{\mathbf{2}} \times \mathbf{Y}$ is algebraically isomorphic to the problem of finding explicit models of D5-branes in $\mathbf{T}^{\mathbf{2}} \times \mathbf{T}^{\mathbf{2}} \times \mathbf{C} / \mathbf{Z}_{\mathbf{N}}$. In [51] explicit models of D5-branes leading to the chiral spectrum of just the Standard Model were constructed. Using the above mapping, it is an straightforward exercise to describe explicit configurations of D6-branes on 3-cycles producing exactly such spectrum.

For instance, consider the configuration of D5-branes with wrapping numbers and Chan-Paton factors as in table 4 in [51] for the particular choice $n_{a}^{1}=n_{d}^{1}=1, \epsilon=$ $\tilde{\epsilon}=\epsilon_{h}=1, \beta^{1}=1 / 2$. This translates into a configuration of D6-branes in a geometry $\mathbf{T}^{\mathbf{2}} \times \mathbf{T}^{\mathbf{2}} \times \mathbf{Y}_{\mathbf{3}}$, with $\mathbf{Y}_{\mathbf{3}}$ having three branch points. The configuration contains D6branes wrapped on the 3 -cycles as follows 25

$$
\begin{array}{rrrl}
a & 3(1,1 / 2) \times(3,-1 / 2) \times \gamma_{0,1} \\
b & 2(2,0) \times(1,-1 / 2) \times \gamma_{1,2} \\
c & 1(2,0) \times(0,1) \times \gamma_{1,2} \\
c & 1(1,-3 / 2) \times(1,-1 / 2) \times \gamma_{0,1} \\
d & & \\
h & 4(2,0) & \times(2,0) \times \gamma_{0,1}
\end{array}
$$

and their orientifold images (denoted $a^{*}, b^{*}$, etc). The configuration is schematically shown in figure 23a).

The configuration can be checked to be free of tadpoles. Upon computation of intersection numbers, we obtain

$$
I_{a b}=1 \quad ; \quad I_{a b^{*}}=2
$$

\footnotetext{
${ }^{25}$ In order to obtain final intersection numbers in agreement with those in [3], we have exchanged $d \leftrightarrow d^{*}$ with respect to 51 .
} 


$$
\begin{array}{rll}
I_{a c}=-3 & ; & I_{a c^{*}}=-3 \\
I_{b d}=0 & ; & I_{b d^{*}}=-3 \\
I_{c d}=-3 & ; & I_{c d^{*}}=-3
\end{array}
$$

precisely reproducing the Standard Model intersection numbers (2.3) in [3]. It should be obvious how to map any other D5-brane model in 51 to a local configuration of D6-branes on 3-cycles with intersection numbers (5).

There is one last issue, crucial to indeed obtain the Standard Model spectrum. This is the constraint that a linear combination of $U(1)$ 's remaining massless plays the role of hypercharge. Very remarkably it is possible to compute the $B F$ couplings in the setup of D6-branes on 3-cycles and recover the same condition as in models of D5-branes.

Hence the above setup provides a new realization of the Standard Model intersection numbers, now in terms of D6-branes wrapped on compact 3-cycles in a non-compact threefold. Although the background geometry is non-supersymmetric, we find the result very satisfactory. The final spectrum is given in table 1, taken from [3].

\begin{tabular}{|c|c|c|c|c|c|c|c|}
\hline Intersection & Matter fields & & $Q_{a}$ & $Q_{b}$ & $Q_{c}$ & $Q_{d}$ & $\mathrm{Y}$ \\
\hline \hline$(\mathrm{ab})$ & $Q_{L}$ & $(3,2)$ & 1 & -1 & 0 & 0 & $1 / 6$ \\
\hline$\left(\mathrm{ab}^{*}\right)$ & $q_{L}$ & $2(3,2)$ & 1 & 1 & 0 & 0 & $1 / 6$ \\
\hline$(\mathrm{ac})$ & $U_{R}$ & $3(\overline{3}, 1)$ & -1 & 0 & 1 & 0 & $-2 / 3$ \\
\hline$\left(\mathrm{ac}^{*}\right)$ & $D_{R}$ & $3(\overline{3}, 1)$ & -1 & 0 & -1 & 0 & $1 / 3$ \\
\hline$\left(\mathrm{bd}^{*}\right)$ & $L$ & $3(1,2)$ & 0 & -1 & 0 & -1 & $-1 / 2$ \\
\hline$(\mathrm{cd})$ & $E_{R}$ & $3(1,1)$ & 0 & 0 & -1 & 1 & 1 \\
\hline$\left(\mathrm{cd}^{*}\right)$ & $N_{R}$ & $3(1,1)$ & 0 & 0 & 1 & 1 & 0 \\
\hline
\end{tabular}

Table 1: Standard model spectrum and $U(1)$ charges

\subsection{Mirror symmetry}

The above intricate agreement in the topological properties of D5-branes at $\mathbf{C} / \mathbf{Z}_{\mathbf{N}}$ and D6-branes in $\mathbf{Y}$ demands a deeper explanation. We strongly suspect that this explanation arises from mirror symmetry between $\mathbf{C} / \mathbf{Z}_{\mathbf{N}}$ and $\mathbf{Y}$, as we roughly explain.

The mirror of $\mathbf{C} / \mathbf{Z}_{\mathbf{N}}$ has been determined using the world-sheet techniques of [53] in 54]. It is described by (the infrared limit of) an $\mathcal{N}=2$ supersymmetric two- 
dimensional Landu-Ginzburg model with superpotential

$$
w(x)=x^{N}+f(x)
$$

where $x$ is a chiral multiplet, and $f(x)$ contains lower order terms which deform the structure of the vacua (and are related to the amount of condensation of closed string twisted tachyons). Its form will not be too important for most of our discussion.

From the geometric viewpoint, the mirror is a non-compact one complex dimensional space $\tilde{\mathbf{Y}}$ described as the hypersurface (5.11) in the $\mathbf{C}^{\mathbf{2}}$ parametrized by $w, x$. It is an $N$-fold cover of the $w$ plane, with $N-1$ critical points $w_{*, i}$ at which $d w / d x=0$. For each value of $w$ there exist $N$ values of $x$ which are pre-images of the map $w(x)$ and so project down to the same point. These values of $x$ are distinct, except at the cricical values $w_{*, i}$, where two pre-images coincide 26. Hence as $w$ varies (5.11) describes $N$ generically disconneted branches, a pair of which touch at each critical point (which pairs join at which critical point depends on the structure of $w)$. Asymptotically at large $w$ the $k^{\text {th }}$ branch tends to $x=w^{1 / N} e^{2 \pi i k / N}$.

The structure of the 1-cycles on which D-branes can wrap in $\tilde{\mathbf{Y}}$ has been determined in [14] (section 5.2). They correspond to real curves coming in from infinity along $x=$ $w^{1 / N} e^{2 \pi i k / N}$, reaching a critical value where the $k^{\text {th }}$ and $k^{\text {th }}$ branches touch, and going off to infinity along $x=w^{1 / N} e^{2 \pi i k^{\prime} / N}$. Such 1-cycle, denoted $\gamma_{\left(k, k^{\prime}\right)}$, has a projection in the $x$-plane which looks like a wedge of angle $2 \pi\left(k-k^{\prime}\right) / N$. Roughly speaking 27 a basis of 1 -cycles is provided by $\tilde{\gamma}_{(i, i+1)}$ with $i=1, \ldots, N$ with the homological relation $\sum_{i=1}^{N} \tilde{\gamma}_{(i, i+1)}=0$, see figure 24c.

The intersection numbers of these 1-cycles 28 , has been computed in [14], sections 2.4 .1 and 5.2 to be

$$
\gamma_{i-1, i} \cdot \gamma_{i, i+1}=1
$$

This precisely reproduces the quiver diagram of the $\mathbf{C} / \mathbf{Z}_{\mathbf{N}}$ orbifold, as expected from mirror symmetry.

There is a remarkable analogy between the structure of 1-cycles in the geometry $\tilde{\mathbf{Y}}$ and our onefolds $\mathbf{Y}$. This relation seems to go beyond mere topology, and hold

\footnotetext{
${ }^{26}$ Such values of $x$ correspond to the (generically massive) vacua of the two-dimensional world-sheet theory.

${ }^{27}$ Which 1-cycles indeed exist depends on the critical point structure and hence on $W$. We assume a suitable choice so that the following basis exists.

${ }^{28}$ Since the 1-cycles are non-compact, the intersection numbers are more properly defined as the number of solitons between the two corresponding two-dimensional vacua of the LG theory.
} 
a)

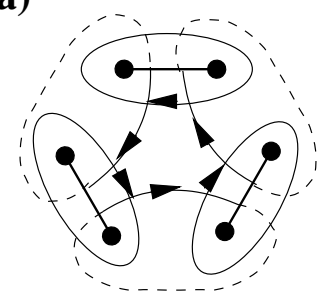

b)

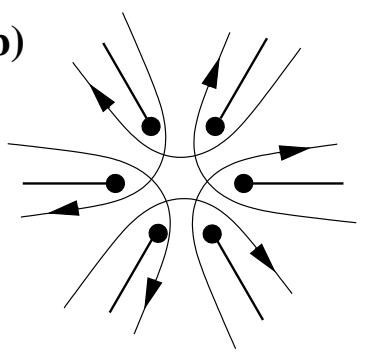

c)

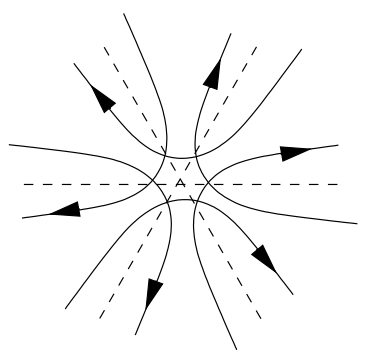

Figure 24: Schematic depiction of the relation between 1-cycles in the geometries $\mathbf{Y}, \tilde{\mathbf{Y}}$. Starting from 1-cycles in $\mathbf{Y}$, one may deform the branch cuts to reach b). Since the 1-cycles in b) do not cross any branch cuts, the double cover structure is not too relevant, and the 1-cycle geometry reproduces that of 1-cycles in $\tilde{\mathbf{Y}}$, figure c.

even at the level of the holomorphic structure, since the geometries may be related by a transformation preserving it, as we show in figure 24. Hence, we strongly suspect that there is a weak equivalence relation between the geometries $\tilde{\mathbf{Y}}$, $\mathbf{Y}$, in the sense of [53, which allows to consider $\mathbf{Y}$ as the mirror of $\mathbf{C} / \mathbf{Z}_{\mathbf{N}}$. This relation would therefore explain the close relation of our D6-brane models in this section to models of D5-branes.

\subsection{Relation to models on $\mathrm{T}^{6}$ with infinite throats}

It is interesting to compare the configurations with Standard Model intersection numbers, realized in terms of D6-branes on $\mathbf{T}^{\mathbf{2}} \times \mathbf{T}^{\mathbf{2}} \times \mathbf{T}^{\mathbf{2}}$ vs on $\mathbf{T}^{\mathbf{2}} \times \mathbf{T}^{\mathbf{2}} \times \mathbf{Y}$. One main difference is that the latter are non-compact geometries; hence (once plugged into a global compact model) allow to lower the string scale while maintaining a large Planck scale, and preventing the appearance of too light Kaluza-Klein gauge bosons. Interestingly enough, in the case discussed above of $\mathbf{Y}$ with three branch points, denote it $\mathbf{Y}_{\mathbf{3}}$, it may be regarded as a two-torus with an infinite throat rendering it non-compact. From this viewpoint, models constructed by gluing $\mathbf{T}^{\mathbf{2}} \times \mathbf{T}^{\mathbf{2}} \times \mathbf{Y}_{\mathbf{3}}$ to a global compact large space may be regarded as models of D6-branes wrapped on a local region which is almost $\mathbf{T}^{\mathbf{6}}$, but which contains a throat connecting it with the rest of (an arbitrarily large) geometry. The models therefore provide an explicit realization of the mechanism proposed in [2] to solve the low string scale problem.

In fact, this suggests an alternative way of constructing Standard Model local configurations of D6-branes on 3-cycles in $\mathbf{T}^{\mathbf{2}} \times \mathbf{T}^{\mathbf{2}} \times \mathbf{Y}_{\mathbf{3}}$. Namely, one may take any model in table 2 in [3], and map the homology class of the 1-cycles in one of the tilted 2-tori 
(by [4] there must be at least one) to homology 1-cycles in $\mathbf{Y}_{\mathbf{3}}$, via the rule

$$
[a] \rightarrow\left[\gamma_{(1,2)}\right] \quad ; \quad[b] \rightarrow\left[\gamma_{(1,2)}\right]-\left[\gamma_{2,0}\right]
$$

This maps consistently the orientifold action (5.6) on 1-cycles in $\mathbf{Y}_{\mathbf{3}}$ with with the orientifold action $[b] \rightarrow-[b],[a] \rightarrow[a]-[b]$ for the tilted 2-tori in [3]; the mapping also preserves intersection numbers (modulo an irrelevant overall sign). Using this mapping the models of D6-branes on $\mathbf{T}^{\mathbf{6}}$ can be translated to models of D6-branes on $\mathbf{T}^{\mathbf{2}} \times \mathbf{T}^{\mathbf{2}} \times \mathbf{Y}_{\mathbf{3}}$, preserving the physical properties of the corresponding gauge theory: for instance, the chiral spectrum, or more remarkably the $B F$ couplings of $U(1)$ generators to RR fields. This allows the construction of large classes of models of D6-branes on 3-cycles in $\mathbf{T}^{\mathbf{2}} \times \mathbf{T}^{\mathbf{2}} \times \mathbf{Y}_{\mathbf{3}}$ with just the Standard Model spectrum; we leave the details to the reader.

Hence the above trick provides a systematic way of starting from a purely toroidal $\mathbf{T}^{\mathbf{6}}$ model and mapping it to a model in a geometry with is $\mathbf{T}^{\mathbf{6}}$ with an infinite throat glued to it. More generally, one may consider threefolds which are products of noncompact Riemann surfaces. This is advantageous since it introduce more non-compact dimensions, and facilitates the task of generating large Planck masses from low string scales. The models may easily be constructed by starting with $\mathbf{T}^{\mathbf{6}}$ models and mapping the 1-homology classes on the $\mathbf{T}^{\mathbf{2}}$ factors to homology classes in $\mathbf{Y}_{\mathbf{3}}$ factors. At the price of giving up supersymmetry in the closed string sector (which may be a moderate one, given that we may lower the string scale), one may construct large classes of models which are toroidal in the neighbourhood of the D6-branes, but contain infinite throats only felt by closed string sector modes.

\section{Conclusions}

Our purpose in the present paper has been to point out the existence of large classes of relatively simple non-compact Calabi-Yau threefolds with compact 3-cycles intersecting at points. They may be easily used to construct local model of four-dimensional chiral gauge theory sectors, by wrapping D6-branes on such 3-cycles. We have also provided the basic rules to compute their intersection numbers and contributions to RR tadpoles, and have illustrated diverse model building possibilities with explicit examples, some of them with phenomenologically very appealing spectrum. In fact, by relaxing the constraint of having a supersymmetry preserving geometry, we have suceeded in constructing (moreover, in giving precise rules to construct large classes of) explicit models with just the Standar Model spectrum. 
We would like to emphasize once more that, even though the threefolds we have centered on first appeared in the context of mirror symmetry, the manifolds can be constructed without help from mirror symmetry. In fact, the strategy should be to construct threefolds with interesting structure of intersecting 3-cycles, and interesting configurations of D6-branes wrapped on them, regardless of their mirror symmetry properties.

There are several directions that should be explored in the present setup. A more complete classification of the kind of threefolds with compact 3-cycles would be desirable. Even in the concrete class studied in this paper, a better control over the sets of $(p, q)$ degenerations that are allowed consistently with the Calabi-Yau condition would be desirable (or even if one relaxes beyond supersymmetric geometries). Also, given a particular geometry, it would be useful to find a systematic and efficient way of describing a basic set of compact and non-compact 3-cycles generating the complete (compact and non-compact) 3-homology, in order to allow a more systematic search for consistent D6-brane configurations, and a systematic computation of their generic spectra. Such formal developments seem quite crucial in providing a general view on the class of gauge theories which may be engineered in this setup, and to search for particular solutions with specific spectra (for instance, the Standard Model intersection numbers in [3]). We hope to report on these ideas in the future.

A final open avenue which we would like to mention is the M-theory lift of configurations of this kind preserving $\mathcal{N}=1$ supersymmetry. As discussed in e.g. 555, they should correspond to purely geometrical configurations involving a non-compact 7-manifold admitting a $G_{2}$ holonomy metric. The possibility of obtaining non-abelian gauge interactions and chiral fermions from such $G_{2}$ configurations had been noticed in [56], and proposed to be used for phenomenological purposes. Unfortunately, compact $G_{2}$ manifolds are difficult to construct, beyond the implicit definition as lifts of IIA configurations with O6-planes and D6-branes [57, 6, 58]. On the other hand, the only known non-compact $G_{2}$ manifolds lead to non-localized gauge interactions, corresponding to A-D-E singularities fibered over non-compact 3-manifolds [56].

The kind of type IIA configurations we have studied, however, would lead to completely four-dimensional gauge interactions and chiral fermions, despite the fact that the complete $G_{2}$ manifold is non-compact. Some of the type IIA configurations we have studied, like that in section 2.5.1, seem symmetric enough to have a relatively simple lift. Although their $G_{2}$ metrics are presumably beyond present techniques (even the metric for the base Calabi-Yau is not known), the topology of the seven-manifold 
is likely to be computable and lead to a simple answer. Such a result would be interesting to e.g. reinterpret tadpole cancellation constraints in terms of geometry (a related discussion is in [59]); but more importantly would provide the first realization of four-dimensional chiral gauge theories genuinely from M-theory on $G_{2}$ manifolds.

\section{Acknowledgements}

I thank L. E. Ibáñez for useful discussions. I also thank M. González for kind support and encouragement.

\section{References}

[1] R. Blumenhagen, L. Goerlich, B. Kors, D. Lust, 'Noncommutative compactifications of type I strings on tori with magnetic background flux', JHEP 0010 (2000) 006, hep-th/0007024.

[2] G. Aldazabal, S. Franco, L. E. Ibanez, R. Rabadan, A. M. Uranga, 'D=4 chiral string compactifications from intersecting branes', J. Math. Phys. 42 (2001) 3103, hep-th/0011073; 'Intersecting brane worlds', JHEP 0102 (2001) 047, hepph/0011132.

[3] L. E. Ibanez, F. Marchesano, R. Rabadan, 'Getting just the standard model at intersecting branes', JHEP 0111 (2001) 002, hep-th/0105155.

[4] R. Blumenhagen, B. Kors, D. Lust, 'Type I strings with F flux and B flux', JHEP 0102 (2001) 030, hep-th/0012156.

[5] S. Forste, G. Honecker, R. Schreyer, 'Orientifolds with branes at angles', JHEP 0106 (2001) 004, hep-th/0105208;

R. Blumenhagen, B. Kors, D. Lust, T. Ott, 'The standard model from stable intersecting brane world orbifolds', Nucl. Phys. B616 (2001) 3, hep-th/0107138;

D. Cremades, L. E. Ibanez, F. Marchesano, 'SUSY Quivers, Intersecting Branes and the Modest Hierarchy Problem', hep-th/0201205;

D. Cremades, L. E. Ibanez, F. Marchesano, 'Intersecting brane models of particle physics and the Higgs mechanism', JHEP 0207 (2002) 022, hep-th/0203160;

C. Kokorelis, 'GUT model hierarchies from intersecting branes', hep-th/0203187;

'New standard model vacua from intersecting branes', hep-th/0205147; 'Exact standard model compactifications from intersecting branes', hep-th/0206108. 
[6] M. Cvetic, G. Shiu, A. M. Uranga, 'Chiral four-dimensional N=1 supersymmetric type 2A orientifolds from intersecting D6 branes', Nucl. Phys. B615 (2001) 3, hep-th/0107166; 'Three family supersymmetric standard - like models from intersecting brane worlds', Phys. Rev. Lett. 87 (2001) 201801, hep-th/0107143.

[7] R. Blumenhagen, V. Braun, B. Körs, D. Lüst, 'Orientifolds of K3 and Calabi-Yau manifolds with intersecting D-branes', JHEP 0207 (2002) 026, hep-th/0206038.

[8] R. Blumenhagen, L. Gorlich, B. Kors, 'Supersymmetric 4-D orientifolds of type IIA with D6-branes at angles', JHEP 0001 (2000) 040, hep-th/9912204;

S. Forste, G. Honecker, R. Schreyer, 'Supersymmetric Z(N) x Z(M) orientifolds in 4-D with D branes at angles', Nucl. Phys. B593 (2001) 127, hep-th/0008250.

[9] S. Kachru, J. McGreevy, 'Supersymmetric three cycles and supersymmetry breaking', Phys. Rev. D61 (2000) 026001, hep-th/9908135;

S. Kachru, S. Katz, A. E. Lawrence, J. McGreevy, 'Open string instantons and superpotentials', Phys. Rev. D62 (2000) 026001, hep-th/9912151; 'Mirror symmetry for open strings' Phys. Rev. D62 (2000) 126005, hep-th/0006047.

[10] H. Ooguri, Y. Oz, Z. Yin, 'D-branes on Calabi-Yau spaces and their mirrors', Nucl. Phys. B477 (1996) 407, hep-th/9606112.

[11] C. Bachas, 'A Way to break supersymmetry', hep-th/9503030.

[12] C. Angelantonj, I. Antoniadis, E. Dudas, A. Sagnotti, 'Type I strings on magnetized orbifolds and brane transmutation', Phys. Lett. B489 (2000) 223, hepth/0007090.

[13] A. Strominger, S. -T. Yau, E. Zaslow, 'Mirror symmetry is T duality', Nucl. Phys. B479 (1996) 243, hep-th/9606040.

[14] K. Hori, A. Iqbal, C. Vafa, 'D-branes and mirror symmetry', hep-th/0005247.

[15] A. Hanany, A. Iqbal, 'Quiver theories from D6 branes via mirror symmetry', JHEP 0204 (2002) 009, hep-th/0108137.

[16] F. Cachazo, B. Fiol, K. A. Intriligator, S. Katz, C. Vafa, 'A Geometric unification of dualities', Nucl. Phys. B628 (2002) 3, hep-th/0110028.

[17] B. Feng, A. Hanany, Y.-H. He, A. Iqbal, 'Quiver theories, soliton spectra and Picard-Lefschetz transformations', hep-th/0206152. 
[18] T. Hauer, A. Iqbal, 'Del Pezzo surfaces and affine seven-brane backgrounds', JHEP 0001 (2000) 043, hep-th/9910054; K. Mohri, Y. Ohtake, S.-K. Yang, 'Duality between string junctions and D-branes on Del Pezzo surfaces', Nucl. Phys. B595 (2001) 138, hep-th/0007243.

[19] H. Ooguri, C. Vafa, 'Geometry of $\mathrm{N}=1$ dualities in four-dimensions', Nucl. Phys. B500 (1997) 62, hep-th/9702180.

[20] K. Ito, 'Seiberg's duality from monodromy of conifold singularity', Phys. Lett. B457 (1999) 285, hep-th/9903061.

[21] C. E. Beasley, M. R. Plesser, 'Toric duality is Seiberg duality', JHEP 0112 (2001) 001, hep-th/0109053;

B. Feng, A. Hanany, Y. -H. He, A. M. Uranga, 'Toric duality as Seiberg duality and brane diamonds', JHEP 0112 (2001) 035, hep-th/0109063.

[22] B. Fiol, 'Duality cascades and duality walls', hep-th/0205155

[23] S. Franco, A. Hanany, 'Geometric dualities in 4-D field theories and their 5-D interpretation', hep-th/0207006.

[24] D. Berenstein, M. R. Douglas, 'Seiberg duality for quiver gauge theories', hepth/0207027.

[25] A. Sen, B. Zwiebach, 'Stable nonBPS states in F theory', JHEP 0003 (2000) 036, hep-th/9907164.

[26] O. DeWolfe, A. Hanany, A. Iqbal, E. Katz, 'Five-branes, seven-branes and fivedimensional E(n) field theories', JHEP 9903 (1999) 006, hep-th/9902179.

[27] M. R. Gaberdiel, B. Zwiebach, 'Exceptional groups from open strings', Nucl. Phys. B518 (1998) 151, hep-th/9709013; M. R. Gaberdiel, T. Hauer, B. Zwiebach, 'Open string-string junction transitions', Nucl. Phys. B525 (1998) 117, hep-th/9801205.

[28] O. DeWolfe, B. Zwiebach, 'String junctions for arbitrary Lie algebra representations', Nucl. Phys. B541 (1999) 509, hep-th/9804210.

[29] O. DeWolfe, 'Affine Lie algebras, string junctions and seven-branes', Nucl. Phys. B550 (1999) 622, hep-th/9809026. 
[30] O. DeWolfe, T. Hauer, A. Iqbal, B. Zwiebach, 'Uncovering the symmetries on $[\mathrm{p}, \mathrm{q}]$ seven-branes: Beyond the Kodaira classification', Adv. Theor. Math. Phys. 3 (1999) 1785, hep-th/9812028.

[31] O. DeWolfe, T. Hauer, A. Iqbal, B. Zwiebach, 'Uncovering infinite symmetries on [p, q] 7-branes: Kac-Moody algebras and beyond' Adv. Theor. Math. Phys. 3 (1999) 1835, hep-th/9812209.

[32] T. Hauer, A. Iqbal, B. Zwiebach, 'Duality and Weyl symmetry of 7-brane configurations', JHEP 0009 (2000) 042, hep-th/0002127.

[33] A. Hanany, E. Witten, 'Type IIB superstrings, BPS monopoles, and threedimensional gauge dynamics', Nucl. Phys. B492 (1997) 152, hep-th/9611230.

[34] M. Berkooz, M. R. Douglas, R. G. Leigh, 'Branes intersecting at angles', Nucl. Phys. B480 (1996) 265, hep-th/9606139.

[35] R. C. McLean, 'Deformations and moduli of calibrated submanifolds', Ph. D. Thesis, Duke University 1990; N. Hitchin, 'The moduli space of special lagrangian submanifolds', math.dg/9711002; 'Lectures on special lagrangian submanifolds', math.dg/9907034.

[36] G. Aldazabal, D. Badagnani, L. E. Ibáñez, A. M. Uranga, 'Tadpole versus anomaly cancellation in D =4, D = 6 compact IIB orientifolds' JHEP 9906 (1999) 031, hep-th/9904071; M. Bianchi, J. F. Morales, 'Anomalies and tadpoles', JHEP 0003 (2000) 030, hep-th/0002149.

[37] M. R. Douglas, G. W. Moore, 'D-branes, quivers, and ALE instantons', hepth/9603167.

[38] M. R. Douglas, B. R. Greene, D. R. Morrison, 'Orbifold resolution by D-branes', Nucl. Phys. B506 (1997) 84, hep-th/9704151.

[39] S. Kachru, E. Silverstein, '4-D conformal theories and strings on orbifolds', Phys. Rev. Lett. 80 (1998) 4855, hep-th/9802183.

[40] A. E. Lawrence, N. Nekrasov, C. Vafa, 'On conformal field theories in fourdimensions', Nucl. Phys. B533 (1998) 199, hep-th/9803015.

[41] A. M. Uranga, 'From quiver diagrams to particle physics', hep-th/0007173. 
[42] A. Hanany, A. M. Uranga, 'Brane boxes and branes on singularities', JHEP 9805 (1998) 013, hep-th/9805139.

[43] L. E. Ibanez, R. Rabadan, A. M. Uranga, 'Anomalous U(1)'s in type I and type IIB D $=4, \mathrm{~N}=1$ string vacua', Nucl. Phys. B542 (1999) 112, hep-th/9808139.

[44] G. Aldazabal, L. E. Ibáñez, F. Quevedo, A. M. Uranga, 'D-branes at singularities: A Bottom up approach to the string embedding of the standard model', JHEP 0008 (2000) 002, hep-th/0005067.

[45] A. M. Uranga, 'D-brane, fluxes and chirality', JHEP 0204 (2002) 016, hepth/0201221.

[46] L. F. Alday, G. Aldazabal, 'In quest of just the standard model on D-branes at a singularity', JHEP 0205 (2002) 022, hep-th/0203129.

[47] S. Schoen, J. fur Math. 364 (1986) 85; M. Bershadsky, V. Sadov, C. Vafa, 'DStrings on D-Manifolds', Nucl. Phys. B463 (1996) 398, hep-th/9510225; C. Vafa, 'Evidence for F-theory', Nucl. Phys. B469 (1996) 403, hep-th/9602022.

[48] S. Mukhopadhyay, K. Ray, 'Fractional branes on a non-compact orbifold', JHEP 0107 (2001) 007, hep-th/0102146;

X. de la Ossa, B. Florea, H. Skarke, 'D-branes on noncompact Calabi-Yau manifolds: K theory and monodromy', hep-th/0104254.

[49] S. Sugimoto, 'Anomaly cancellations in type I D-9 - anti-D-9 system and the USp(32) string theory', Prog. Theor. Phys. 102 (1999) 685, hep-th/9905159;

I. Antoniadis, E. Dudas, A. Sagnotti, 'Brane supersymmetry breaking', Phys. Lett. B464 (1999) 38, hep-th/9908023;

G. Aldazabal, A. M. Uranga, 'Tachyon free nonsupersymmetric type IIB orientifolds via Brane - anti-brane systems', JHEP 9910 (1999) 024, hep-th/9908072.

[50] J. Lykken, E. Poppitz, S. P. Trivedi, 'Branes with GUTs and supersymmetry breaking', Nucl. Phys. B543 (1999) 105, hep-th/9806080.

[51] D. Cremades, L. E. Ibanez, F. Marchesano, 'Standard model at intersecting D5branes: Lowering the string scale', hep-th/0205074.

[52] D. Bailin, G. V. Kraniotis, A. Love, 'Standard - like models from intersecting D4branes', Phys. Lett. B530 (2002) 202, hep-th/0108131; H. Kataoka, M. Shimojo, 
' $S U(3) \times S U(2) \times U(1)$ chiral models from intersecting D4-branes / D5-branes', hep-th/0112247; G. Honecker, 'Intersecting brane world models from D8-branes on $\mathbf{T}^{\mathbf{2}} \times \mathbf{T}^{\mathbf{4}} / \mathbf{Z}_{\mathbf{3}} / \mathbf{\Omega} \mathbf{R}_{\mathbf{1}}$ type IIA orientifolds', JHEP 0201 (2002) 025, hep-th/0201037; C. Kokorelis, 'Exact standard model structures from intersecting d5-branes', hepth/0207234.

[53] K. Hori, C. Vafa, 'Mirror symmetry', hep-th/0002222.

[54] C. Vafa, 'Mirror symmetry and closed string tachyon condensation', hepth/0111051.

[55] J. Gomis, 'D-branes, holonomy and M theory', Nucl. Phys. B606 (2001) 3, hepth/0103115.

[56] M. Atiyah, E. Witten, 'M theory dynamics on a manifold of G(2) holonomy', hepth/0107177;

B. Acharya, E. Witten, 'Chiral fermions from manifolds of G(2) holonomy', hepth/0109152.

[57] S. Kachru, J. McGreevy, 'M theory on manifolds of G(2) holonomy and type IIA orientifolds', JHEP 0106 (2001) 027, hep-th/0103223.

[58] M. Cvetic, G. Shiu, A. M. Uranga, 'Chiral type II orientifold constructions as M theory on G(2) holonomy spaces', hep-th/0111179.

[59] E. Witten, 'Anomaly cancellation on G(2) manifolds', hep-th/0108165. 